\title{
Single-cell epigenetic analysis reveals principles of chromatin states in H3.3-K27M gliomas
}

Nofar Harpaz ${ }^{1+}$, Tamir Mittelman ${ }^{1+}$, Olga Beresh ${ }^{1}$, Tomer-Meir Salame ${ }^{2}$, Noa Furth ${ }^{1}$, Guy Ron ${ }^{3}$, Efrat Shema ${ }^{1 *}$.

${ }^{1}$ Department of Biological Regulation, Weizmann Institute of Science, Rehovot 76100, Israel.

${ }^{2}$ Flow Cytometry Unit, Life Sciences Core Facilities, Weizmann Institute of Science, Rehovot 76100, Israel

${ }^{3}$ Racah Institute of Physics, Hebrew University, Jerusalem 91904, Israel.

* Corresponding author:

Efrat.shema@,weizmann.ac.il

${ }^{+}$Equal contribution 


\begin{abstract}
Cancer cells are highly heterogeneous, both at the transcriptional level and in their epigenetic state. Methods to study epigenetic heterogeneity are limited in their throughput and the information obtained per cell. Here, we adapted Cytometry by Time of Flight (CyTOF) to analyze a wide panel of histone modifications and chromatin regulators in primary tumor-derived lines of Diffused Intrinsic Pontine Glioma (DIPG). DIPG is a lethal pediatric brain cancer, driven by a mutation in histone H3 leading to substitution of lysine 27 with methionine (H3-K27M mutation). We identified two epigenetically distinct subpopulations in DIGP, reflecting inherent heterogeneity in the expression levels of the mutant histone. These two epigenetic subpopulations are robust across tumor lines derived from different patients and show differential proliferation capacity as well as expression of stem-cell and differentiation markers. Moreover, we demonstrate the use of this single-cell high-dimensional data to elucidate potential interactions between histone modifications and epigenetic alterations during the cell-cycle. Our work establishes new concepts for the analysis of epigenetic heterogeneity and cross-talk in cancer that could be applied to diverse biological systems.
\end{abstract}

\title{
Introduction
}

Epigenetic regulation of genome function is fundamental for cellular differentiation, and its deregulation often promotes cancer ${ }^{1-3}$. Post-translational modifications (PTMs) of histones, which compose the nucleosome structure, regulate gene transcription by mediating interactions with the transcriptional machinery and by physically affecting DNA accessibility. In the last decade, singlecell RNA sequencing technologies have revolutionized our understanding of transcriptional heterogeneity within normal and malignant tissues ${ }^{4,5}$. Parallel techniques were developed to shed light on epigenetic cellular heterogeneity, mainly focusing on single-cell DNA methylation and chromatin accessibility $^{6-10}$. However, the plethora of histone PTMs is mainly analyzed using bulk methods such as Chromatin Immunoprecipitation and sequencing (ChIP-seq). Recently, single-cell ChIP-seq and Cut\&Tag revealed the patterns and heterogeneity of several histone modifications in the mouse brain, during stem cell differentiation, and in breast cancer ${ }^{11-14}$. Advances in optical microscopy and super-resolution imaging allowed single-cell analysis of histone modifications and their spatial localization in the nucleus ${ }^{15-17}$. While these advances show great promise, they are all limited in scale and throughput, allowing analysis of one or two modifications at a time per single cell. Recently, a single-cell approach, based on Cytometry by Time of Flight (CyTOF), was used 
to study the epigenome of blood immune cells, demonstrating epigenetic alterations occurring with $\operatorname{age}^{18}$, and following vaccination ${ }^{19}$.

Mutations in epigenetic regulators, as well as in histone proteins, are frequent in many types of cancer. One striking example is the lysine 27 mutation of histone H3, leading to its substitution with methionine (H3-K27M), which is the driving event for Diffused Intrinsic Pontine Glioma (DIPG). DIPG is a lethal, incurable, pediatric cancer, usually diagnosed in children aged 5-10. More than $80 \%$ of cases show a H3-K27M mutation on one allele out of 32 that encode canonical histone $\mathrm{H} 3.1$ or the histone variant $\mathrm{H} 3.3^{20-24}$. This gain-of-function mutation was shown to cause drastic alterations in the epigenome, in both cis (on the mutant histones themselves) and trans (epigenetic alterations to wild-type histones) ${ }^{25}$. The most notable changes induced by H3-K27M are the global loss of the repressive modification tri-methylation of histone H3 on lysine 27 (H3K27me3), with concomitant gain in acetylation of that same lysine residue (H3K27ac) $)^{26-30}$.

Here, we propose a novel adaptation and analysis of CyTOF, with a unique custom-designed epigenetic panel, as a method to reveal epigenetic dynamics and heterogeneity in cancer. We demonstrate the power of this method in revealing: (1) Systematic effects of chromatin perturbations, such as the H3-K27M mutation in glioma; (2) Discovery of epigenetic heterogeneity within tumor lines, as exemplified by two distinct subpopulations in DIPG; (3) Identification of baseline correlations between chromatin modifications, suggesting mechanisms of co-regulation; and (4) Characterization of dynamic epigenetic processes in single cells. This high-dimensional single-cell data is instrumental in deducing principles of epigenetic heterogeneity and regulation in cancer.

\section{Results}

\section{CyTOF Epi-Panel reveals global epigenetic alterations induced by the K27M-mutant histone}

To explore CyTOF as a method to reveal systematic effects of chromatin perturbations in cancer, we applied it to examine glioma models expressing the mutant histone H3.3-K27M. We designed an epigenetics-oriented panel, containing antibodies targeting the H3-K27M histone mutant, 16 well-characterized histone modifications, 5 histones proteins (H1, H1.0, H3, H3.3 and H4) and 3 chromatin regulators. Furthermore, we extended this panel with oncogenic, cancer stem cell and glioma lineage markers, as well as chromatin regulators and cell-cycle specific indicators (Figure 1A). Each selected antibody was conjugated to a specific metal, while taking into consideration 
several parameters in metal allocation to enable parallel reading of all the antibodies in the same cells and minimize technical noise (see Methods).

We first applied this epigenetic panel to an isogenic system of HEK293 cells with inducible expression of either wild type (WT) histone H3.3 or the H3.3-K27M-mutant histone. To minimize batch effect, samples were initially barcoded so that subsequent processing and CyTOF runs could be done simultaneously. To validate the system, we performed CyTOF experiments on three independent biological repeats, showing very high reproducibility between experiments (Figure 1B-C, S1A-B). We obtained single-cell measurements from a total of 72,316 and 76,695 cells expressing the WT or K27M-mutant histone, respectively. Each measurement of histone modification was normalized to the level of core histones H3, H4, and H3.3 in that cell (see Methods for details on the normalization method), followed by dimension reduction Uniform Manifold Approximation and Projection (UMAP) ${ }^{31}$ analysis. Interestingly, HEK293 cells expressing the mutant H3.3 clustered separately from the same cells expressing the WT histone, using solely histone modifications for this algorithm and excluding the H3-K27M measurements (Figure 1B). This suggests that expression of H3-K27M results in global epigenetic alterations. Of note, we did detect a small fraction of the H3-K27M mutant cells that clustered with the WT cells. A closer examination of these cells revealed background levels of H3-K27M measurements, indicating that they had failed to express the H3-K27M construct, and were thus removed from farther analysis (Figure S1C).

The most prominent epigenetic alterations induced by expression of H3-K27M were the global loss of $\mathrm{H} 3 \mathrm{~K} 27 \mathrm{me} 3$ and gain in $\mathrm{H} 3 \mathrm{~K} 27 \mathrm{ac}$, as is well documented in the literature ${ }^{26-29}$ (Figure 1B-C, S1D). In addition to these known effects, the extended panel revealed alterations in many other epigenetic modifications, associated with both active and repressed chromatin states. Concomitant with the increase in H3K27ac, we observed a marked increase in lysine 16 acetylation on histone $\mathrm{H} 4$ (H4K16ac). In addition, the data revealed a global increase in the activating marks mono- and tri-methylation of histone $\mathrm{H} 3$ on lysine $4(\mathrm{H} 3 \mathrm{~K} 4 \mathrm{me} 1, \mathrm{H} 3 \mathrm{~K} 4 \mathrm{me} 3)$ as well as lysine 9 and 64 acetylations (H3K64ac, H3K9ac), albeit at a lower magnitude. These alterations suggest a more 'open' chromatin state induced by the H3-K27M mutation. However, in parallel, there is also an increase in the heterochromatin-associated mark tri-methylation of lysine 9 on histone $\mathrm{H} 3$ (H3K9me3), as was recently reported ${ }^{32}$. In addition, we observed a reduction in the mitosisassociated phosphorylation of histone $\mathrm{H} 3$ ( $\mathrm{pH} 3 \mathrm{~S} 28$ ) and in cleaved $\mathrm{H} 3$, a proteolytic form of histone $\mathrm{H} 3$ that is linked with mammalian differentiation ${ }^{33-35}$ (Figure 1C). These results were highly reproducible across independent experiments with different induction times of H3-K27M (4, 7 and 
10 days) (Figure S1A-B), pointing to the robustness of the epigenetic alterations and the quantitative nature of CyTOF in revealing systematic effects mediated by this mutation.

The global increase in histone acetylation, suggesting a general opening of chromatin structure, motivated us to explore the shape of the nuclei in cells expressing the mutant histone. Interestingly, we observed that HEK293 cells expressing H3-K27M harbor significantly larger nuclei than isogenic cells expressing WT H3.3 (Figure 1E-F). To further examine whether the increase in nuclei size is a result of the global increase in histone acetylations, we treated WT HEK293 cells with the histone deacetylase inhibitor Vorinostat (HDACi) for 72 hours. Prolonged HDACi treatment led to a similar increase in nuclei size that was observed upon H3-K27M induction (Figure 1G-H). These results suggest that expression of the K27M-mutant histone results in elevated histone acetylations on several lysine residues, presumably leading to a more 'open' chromatin structure and enlarged nuclei.

\section{Single-cell profiling identifies epigenetic heterogeneity in H3-K27M tumor-derived primary cell lines}

To examine H3-K27M-associated epigenetic alterations in a more biologically relevant system, we applied our CyTOF Epi-Panel to study two patient-derived DIPG cultures: SU-DIPG13, expressing the H3-K27M mutation endogenously, and SU-DIPG48, harboring WT H3. As expected, UMAP analysis of these lines, based solely on epigenetic marks, resulted in separate and distant clustering of each patient (Figure 2A). H3-K27M expression was restricted only to SU-DIPG13 cells, which also showed the expected low levels of H3K27me3 and high H3K27ac. Interestingly, while SUDIPG48 cells clustered to a single center, SU-DIPG13 showed a clear division into two distinct subpopulations (Figure 2A-B, S2A). We repeated the UMAP analysis for SU-DIPG13 cells alone, and trained a gradient boosting algorithm ${ }^{36}$ to determine the epigenetic features that affect the assignment to the two clusters (Figure 2B-C, S2B-D). The results showed these two clusters mainly originated from higher expression of the H3-K27M mutant histone, H3K27ac and H3K4me1 pushing the cells towards one cluster, while increased levels of $\mathrm{H} 3 \mathrm{~K} 9 \mathrm{me} 3$, cleaved $\mathrm{H} 3$ and $\mathrm{pH} 3 \mathrm{~S} 28$ pushed cells towards the second cluster. In fact, the gradient boosting algorithm allocated single cells into the correct cluster at $91 \%$ percent accuracy using the reads of only four modifications: H3K27ac, cleaved H3, H3K9me3 and H3K4me1 (Figure S2D). Intriguingly, although DIPG cells all contain the H3-K27M mutation endogenously, the expression of the mutant gene varies in the population, with some cells expressing it at higher or lower levels. This variability in H3-K27M expression likely induces epigenetic heterogeneity in the population, thus generating the two distinct epigenetic subpopulations we termed H3-K27M-low and H3-K27M-high. 
To characterize this epigenetic heterogeneity identified in SU-DIPG13, we examined fold change differences of all chromatin modifications in these two subpopulations. Similar to the trends observed in the HEK293 inducible system, the H3-K27M-high cluster showed elevation of all active modifications, mainly $\mathrm{H} 3 \mathrm{~K} 27 \mathrm{ac}, \mathrm{H} 4 \mathrm{~K} 16 \mathrm{ac}$ and $\mathrm{H} 3 \mathrm{~K} 4 \mathrm{me} 1 / 3$, and reduced levels of cleaved H3 and pH3S28 (Figure 2B,D). Interestingly, the H3-K27M-low cluster showed higher levels of the facultative heterochromatin mark $\mathrm{H} 3 \mathrm{~K} 9 \mathrm{me} 3$. This result seemed contradictory to our previous data, as H3K9me3 levels increased in the HEK293 cells expressing H3-K27M (Figure 1C), and decreased in SU-DIPG13 cells upon knock-out of the H3-K27M mutant allele ${ }^{32}$ (Figure S2E), suggesting it is positively regulated by H3-K27M expression. However, in the context of the two epigenetic subpopulations, high levels of $\mathrm{H} 3 \mathrm{~K} 9 \mathrm{me} 3$ strongly associated with low expression of $\mathrm{H} 3$ $\mathrm{K} 27 \mathrm{M}$, prompting us to further explore this regulation in a dynamic setup.

\section{CyTOF reveals dynamic epigenetic alterations mediated by $\mathrm{H3}-\mathrm{K} 27 \mathrm{M}$, suggesting various modes of regulation}

To explore the dynamics of epigenetic alterations induced by H3-K27M and shed light on different modes of regulation by low versus high levels of H3-K27M expression, we took advantage of our inducible HEK293 system. We treated these cells with doxycycline to induce expression of H3K27M for 8, 16, 48 and 96 hours, followed by CyTOF with a focused panel of six epigenetic marks, $\mathrm{H} 3-\mathrm{K} 27 \mathrm{M}$, and the core histones for normalization. We observed a gradual increase in the levels of H3-K27M expression, reaching saturation at 48 hours (Figure 3A-C). A highly coordinated behavior was seen for H3K27ac, H4K16ac and H3K36me2. H3K27me3 levels, however, were not affected at the short induction of 8 hours, and started to decrease gradually only at later time points (Figure 3C). This is in line with the high stability of this modification, which is thought to be mainly 'diluted' during cell-cycle progression rather than being actively removed by histone demethylases ${ }^{37,38}$. These results indicate that the increase in histone acetylations occurs prior to loss of $\mathrm{H} 3 \mathrm{~K} 27 \mathrm{me} 3$, and is likely to be a primary effect of $\mathrm{H} 3-\mathrm{K} 27 \mathrm{M}$ expression rather than a secondary effect of the loss of $\mathrm{H} 3 \mathrm{~K} 27 \mathrm{me} 3$.

Contrary to the gradual increase/decrease over time seen for most epigenetic marks, for H3K9me3 and cleaved $\mathrm{H} 3$ there was a complex non-monotonic behavior; at early time points $\mathrm{H} 3-\mathrm{K} 27 \mathrm{M}$ was still moderately expressed $\mathrm{H} 3 \mathrm{~K} 9 \mathrm{me} 3$ and cleaved $\mathrm{H} 3$ expression spiked, however, as $\mathrm{H} 3-\mathrm{K} 27 \mathrm{M}$ expression increased, H3K9me3 and cleaved H3 levels gradually increased (Figure 3D). Interestingly, this behavior reflects our observations in SU-DIPG13, in which higher levels of H3K9me3 and cleaved H3 were detected in the H3-K27M-low cluster. The data supports a complex relationship between $\mathrm{H} 3-\mathrm{K} 27 \mathrm{M}$ expression and $\mathrm{H} 3 \mathrm{~K} 9 \mathrm{me}$, which is highly dependent on the 
relative levels of H3-K27M expression. Moreover, these results provide further evidence for the importance of $\mathrm{H} 3 \mathrm{~K} 9 \mathrm{me} 3$ and cleaved $\mathrm{H} 3$ in dictating the two distinct epigenetic subpopulations in SU-DIPG13.

\section{The two epigenetic subpopulations are robust across different DIPG tumor-derived lines and show distinctive proliferation and differentiation features}

Our findings of epigenetic heterogeneity in SU-DIPG13 prompted us to explore whether this phenomenon was unique to that cell line, or if it could be generalized to additional DIPG tumorderived lines. To that end, we performed the CyTOF experiment in a primary cell line derived from a different patient with a H3.3-K27M mutation: SU-DIPG6. In parallel, we repeated the CyTOF experiment for SU-DIPG13, aiming to examine more cells as well as expand our panel of antibodies (Figure 1A). UMAP analysis showed that both SU-DIPG6 and SU-DIPG13 presented two epigenetic clusters, although the proportion of cells in the H3-K27M-low cluster was lower than in previous experiments ( $\sim 10 \%$ versus $\sim 40 \%$, Figure 4 A, S3A). Moreover, a shared UMAP of both lines showed they clustered together, indicating a highly similar epigenetic behavior, unlike SUDIPG48 expressing WT H3, which clustered separately from SU-DIPG13 (Figure 4B, 2A). Reproducing the previous CyTOF experiment, in both DIPG lines the H3-K27M-low cluster had higher mean levels of cleaved H3, H3K9me3, H3K36me2 and cells expressing pH3S28, while the H3-K27M-high cluster was characterized by higher levels of H3K27ac, H416ac and H4K4me1/3 (Figure 4C-D, S3B,D, S4C). Interestingly, we also observed an increase in EZH2, the catalytic unit of PRC2, in the H3-K27M-high cluster (Figure 4D, S3C,D, S4A,C). This result may reflect a feedback mechanism to the loss of $\mathrm{H} 3 \mathrm{~K} 27 \mathrm{me} 3$, which needs to be further investigated.

The histone variant H1.0 is known to be expressed heterogeneously within tumors, and H1.0 silencing by a fraction of tumor cells has been suggested to facilitate the transcription of oncogenes and stem-cell related genes ${ }^{39}$. We found that H1.0 mean levels were reduced in the H3-K27M-high cluster, and a smaller fraction of cells expressed it in this cluster (Figure 4A,C, E, S3B). Moreover, H1.0 levels decreased in the HEK293 isogenic cells expressing H3-K27M compared to cells expressing WT H3.3, indicating the mutant histone affects H1.0 expression in diverse biological systems (Figure 4F). Interestingly, the cleaved form of $\mathrm{H} 3$, which is associated with cellular differentiation processes ${ }^{33-35}$, also showed reduced levels in the H3-K27M-high cluster, suggesting a lower differentiation state of these cells.

SOX2 and c-Myc are well-known drivers of the stem-cell state, as well as oncogenes in the context of gliomagenesis ${ }^{40,41}$. SOX2 was reported to be induced by the ectopic expression of H3-K27M in 
an oligodendrocyte progenitor cell line, and to play an essential role in inducing a stem-cell-like state in glioblastoma ${ }^{40,41}$. We therefore added these proteins to the CyTOF panel, and saw elevated levels of both SOX2 and c-Myc in the H3-K27M-high cluster (Figure 4G, S3C, S4A).c-Myc was also upregulated in the HEK293 cells expressing H3-K27M (Figure 4F, S4B). In addition, we examined markers for the neural progenitor cells (NPCs) and the mesenchymal-like cells (MESlike) CD24 and CD44, respectively ${ }^{42}$. Unlike SOX2 and c-Myc, most cells did not express either of these cell identity markers. However, upon examination of the fraction of CD24+ or CD44+ cells, we saw more CD24+ cells were enriched in the H3-K27M-high cluster compared to the H3K27M-low cluster, and an opposite trend was observed for CD44+ cells (Figure 4H). Other markers, including the glioblastoma lineage markers CXCR4, GFAP, DLL3 and MBP, and the chromatin regulators SIRT-1 and BMI1, did not behave differently between the clusters (Fig S3C, S4A). The elevated levels of SOX2, c-Myc and CD24 in cells expressing high levels of H3-K27M, in addition to the reduced expression of $\mathrm{H} 1.0$ and cleaved H3, suggest that cells belonging to the H3-K27M-high cluster represent the fraction of tumor cells that had higher self-renewal, stem-celllike properties.

Following the observation that H3-K27M-high cells are enriched with markers indicative of a less differentiated state, we aimed to examine their proliferation capacity. We therefore included in our CyTOF panel the proliferation marker, Ki-67. In both SU-DIPG6 and SU-DIPG13 cells, the H3K27M-high cluster expressed higher levels of Ki-67 when compared to the H3-K27M-low cluster (Figure 4I-J). Of note, despite reduced Ki-67 in the H3-K27M-low cells, a higher fraction of these cells were positive for the mitosis-associated $\mathrm{pH} 3 \mathrm{~S} 28$, perhaps indicating prolonged unproductive mitosis (pH3S28, Figure 4C, S3B,E, S4D). Interestingly, the single-cell analysis revealed H3K27M-mediated dependency between Ki-67 and the oncogenic c-Myc, as the correlation between these two markers increased significantly in the H3-K27M-high cluster (Figure 4K). These results support the notion that the less differentiated H3-K27M-high cells are more proliferative. This is in line with a recent single-cell RNA-seq study of DIPG tumors, showing that the majority of tumor cells are indeed less differentiated, and these are the cells with the higher proliferation capacity that presumably maintain the tumor's aggressive behavior ${ }^{43}$.

\section{Single-cell CyTOF analysis uncovers potential co-regulation of histone modifications}

We next aimed to explore whether CyTOF single-cell multi-parametric data can be leveraged to study baseline correlations between histone modifications, and the effects of epigenetic perturbations on these correlations. Our underlying assumption is that histone modifications that are mechanistically linked (for example, deposited or removed by the same enzymes) should be 
highly correlated at the single-cell level. As we have measurements of multiple repressive and active modifications within the same cells, we might be able to deduce potential cross-talk and interactions between different epigenetic marks. To that end, we calculated the pairwise correlations between all pairs of histone modifications measured by CyTOF (Figure S5A). We first examined all the modifications associated with gene activation: H3K27ac, H3K9ac, H3K64ac, H4K16ac, H3K4me3 and H3K4me1. As shown in Figure 5A and S5A-C, H3 acetylations on lysine 9, 27 and 64 cluster with each other. This result aligns with our hypothesis, as these modifications are known to be deposited by similar enzymatic complexes (EP300/CREBBP ${ }^{44,45}$ ). Of note, lysine 16 acetylation on histone $\mathrm{H} 4$ (H4K16ac), which is deposited by the distinct acetyltransferase Males absent On the First (MOF) ${ }^{46}$, shows lower correlation and does not cluster with $\mathrm{H} 3$ acetylations, further supporting our hypothesis. Interestingly, among the highest correlations were those measured between H4K16ac and the two methylation states of lysine 4 on histone H3 (H3K4me1 and H3K4me3, Figure 5A, S5B-D). In HeLa cells, MOF was shown to form a stable complex with MLL1, and the purified complex had robust methyltransferase activity in depositing H3K4me1$3^{47}$. The high correlation between these modifications in HEK293 cells suggests this complex may be active in diverse cellular systems, generating synchronized deposition of these histone marks. In addition, recent work identified the PHD finger domain of MLL4, the major methyltransferase that deposits $\mathrm{H} 3 \mathrm{~K} 4 \mathrm{me} 1$, as a specific reader of $\mathrm{H} 4 \mathrm{~K} 16 \mathrm{ac}^{48}$.

We next examined the correlations of repressive modifications with each other and with active chromatin marks (Figure 5B, S5E). As expected, H3K27me3 is highly correlated with monoubiquitination of histone $\mathrm{H} 2 \mathrm{~A}$ (H2Aub), reflecting the well-established interaction between Polycomb Repressive Complex 1 (PRC1) that binds H3K27me3 and deposits H2Aub ${ }^{49}$. The H3K9me3 modification, the hallmark of constitutive heterochromatin, is much less correlated with both the facultative heterochromatin modifications $\mathrm{H} 3 \mathrm{~K} 27 \mathrm{me} 3 / \mathrm{H} 2 \mathrm{Aub}$ and the active modifications (H3 acetylations). This observation is in line with the different genomic distributions of these modifications measured by ChIP-seq ${ }^{50}$ and immunofluorescence (Figure 5C). Interestingly, H3K9me3 shows high correlation with the intergenic mark H3K36me2, perhaps reflecting the fact that both are targeted by the same histone demethylase JMJD2 $\mathrm{A}^{51}$. These results point to the potential of single-cell correlation analysis for uncovering co-regulation and mechanistic cross-talk between epigenetic modifications.

To explore how epigenetic perturbation effect these pairwise correlations, we analyzed the HEK293 cells expressing H3-K27M. We observed a general increase in all correlations between open chromatin marks in $\mathrm{H} 3-\mathrm{K} 27 \mathrm{M}$ cells, in line with the notion that this mutation causes a global 
opening of the chromatin structure (Figure 5D). The most prominent increase in correlation is observed between H3K27ac and H4K16ac, the two modifications that are most upregulated by H3K27M. To further generalize these observations, we also explored pairwise correlations in SUDIPG13 and SU-DIPG6 tumor lines endogenously expressing H3-K27M. As expected, H3 acetylations on lysine 9 and lysine 64 are highly correlated and cluster together in these cells, similar to our observations in WT cells. Surprisingly, H3K27ac in the DIPG cells showed a higher correlation with $\mathrm{H} 4 \mathrm{~K} 16 \mathrm{ac}$ rather than $\mathrm{H} 3$ acetylations (Figure 5E, S5F). This result reflects the epigenetic alterations caused by H3-K27M, coupling these two acetylations on histone H3 and H4. It highlights the use of CyTOF data to deduce both baseline correlations between modifications, as well as gain insights on how epigenetic perturbations affect various epigenetic pathways.

\section{Leveraging single-cell data to analyze dynamic chromatin alterations during the cell cycle}

To further explore the utility of CyTOF in revealing dynamic processes within a population, we aimed to leverage this data to more broadly identify global epigenetic alterations associated with S-phase, as marked by 5-Iodo-2'-deoxyuridine (IdU) that is incorporated during DNA synthesis (Figure 6A). The highly proliferative SU-DIPG13 \& 6 lines are ideal for this analysis, due to the high percentage of cells undergoing S-phase. We thus gated the cells into two populations based on the IdU histogram and compared their epigenetic states (Figure 6A-D, S6A-D). We found that the chromatin in S-phase seems to be in a more 'open' state: it is enriched with active marks such as $\mathrm{H} 3$ acetylations, and show reduced levels of the repressive $\mathrm{H} 3 \mathrm{~K} 9 \mathrm{me} 3$ and $\mathrm{H} 2 \mathrm{Aub}$ (Figure 6CD, S6C-D). Interestingly, H4K16ac decreased during S-phase, emphasizing the distinct regulation of $\mathrm{H} 3$ and $\mathrm{H} 4$ acetylations, as also indicated by their lower correlation (Figure 5A-B). In contrast to the previously shown pattern of a global increase in histone methylations ${ }^{52}$, we observed a decrease in most methylations examined, including the transcription-associated mark H3K36me3. Unlike most methylations, H3K4me3 levels increased during S-phase, in agreement with the open chromatin state (Figure 6D, S6D).

Finally, we decided to focus on the IdU-positive population, and leverage our data to explore the epigenetic alterations that occur with time during S-phase. We used the iridium labeling that marks total DNA content to divide the S-phase population accordingly: cells with high iridium, indicating high DNA content, are at a late stage of DNA synthesis, while cells with low iridium are in early S-phase. During DNA synthesis, cells express and synthesize the core histone proteins to enable the formation of nucleosomes on the nascent DNA strand. To validate our approach, we analyzed the raw amounts of core histones (pre-normalization) in the early and late S-phase populations, and saw an increase in $\mathrm{H} 3, \mathrm{H} 4$ and $\mathrm{H} 1$ in late DNA synthesis (Figure 6E). Next, we explored the pattern 
of histone modifications along S-phase, normalizing their levels to the measured levels of the core histones $\mathrm{H} 3$ and $\mathrm{H} 4$ (to account for the total increase in histones expression). As expected, almost all histone modifications showed decreased levels in late S-phase, compatible with the dilution of the parental modified histones and incorporation of new histones that are not yet modified (Figure $6 \mathrm{~F}, \mathrm{~S} 6 \mathrm{E}, \mathrm{F})$. Levels of the repressive modification $\mathrm{H} 3 \mathrm{~K} 9 \mathrm{me} 3$, which were reported to be restored slowly and fully just by the next G1 cycle $^{38}$, showed a prominent decrease in late S-phase. The fold change of $\mathrm{H} 3 \mathrm{~K} 4 \mathrm{me} 3$ in late versus early S-phase is lower than that of $\mathrm{H} 3 \mathrm{~K} 9 \mathrm{me} 3$, indicating a faster restoration of this histone mark, in agreement with previous reports showing complete restoration of $\mathrm{H} 3 \mathrm{~K} 4 \mathrm{me} 3$ in $\mathrm{G}^{53}$. Interestingly, the modifications that showed the fastest restoration were the three $\mathrm{H} 3$ acetylations, with $\mathrm{H} 3 \mathrm{~K} 9 \mathrm{ac}$ even showing a slight increase in late S-phase. These results may reflect the more dynamic nature of histone acetylations versus methylations ${ }^{37}$, and suggest faster kinetics of histone acetyltransferases versus methyltransferases. Moreover, studies of DNA replication timing showed that open, transcriptionally active chromatin is replicated early, while closed chromatin is replicated in late S-phase ${ }^{54}$. This may account for the higher restoration of histone acetylations seen in late S-phase, as well as H3K4me3, compared with close chromatin modifications such as $\mathrm{H} 3 \mathrm{~K} 9 \mathrm{me} 3$. Of note, $\mathrm{H} 4 \mathrm{~K} 16 \mathrm{ac}$ did not present this quick restoration phenotype, suggesting a different regulatory mechanism.

\section{Discussion}

In this study, we present the advantages of the CyTOF methodology to achieve a broad analysis of histone modification states in cancer cells at a single-cell resolution. The H3-K27M mutation in DIPG is known to drive drastic epigenetic alterations, however the extent of these alterations, the epigenetic marks that are influenced by it, and its heterogeneity within the population are unclear. We identified upregulation of H4K16ac as one of the most prominent effects of H3-K27M expression, similar to the well-established upregulation of $\mathrm{H} 3 \mathrm{~K} 27 \mathrm{ac}^{29}$. Schachner et al. recently applied mass spectrometry to intact nucleosomes and showed high levels of H4K16ac on H3K27M-mutant nucleosomes ${ }^{55}$, supporting a cis effect of the mutation on this specific H4 acetylation. The global increase observed in our data strongly suggests a trans effect as well, similar to H3K27ac. Interestingly, our data suggests that these two acetylations, H3K27ac and H4K16ac, are likely to be distinctly regulated; first, they show a lower correlation between themselves compared to the correlations between $\mathrm{H} 3$ acetylations that are known to share enzymatic writers. Second, while H4K16ac is upregulated by H3-K27M, similarly to other acetylations, it shows a distinct behavior during S-phase, where its restoration to newly deposited histones is slow and does 
not follow $\mathrm{H} 3$ acetylations. Finally, studies report that H4K16ac is deposited by a specific acetyltransferase, MOF, which does not target other lysine residues. Collectively, this data suggests unique functions for this acetylation that are not redundant with $\mathrm{H} 3$ acetylations.

The single-cell analysis revealed epigenetic heterogeneity within the DIPG tumor-derived cell lines, generating two distinct epigenetic subpopulations with unique characteristics. Interestingly, these epigenetic subpopulations are associated with inherent heterogeneity in H3-K27M expression, and were not detected in a DIPG line containing WT H3. While H3-K27M is expressed in a gradient across the population, the clear division into two clusters suggests a threshold effect for mediating various epigenetic alterations. In line with this notion is our finding that H3K9me3 and cleaved H3 levels are affected differently by the levels of H3-K27M in individual cells. The heterogeneity in $\mathrm{H} 3-\mathrm{K} 27 \mathrm{M}$ expression itself could also stem from epigenetic changes in its chromatin environment, generating a feedback mechanism that may further stabilize the two distinct states. Interestingly, while these two states are seen in two different patient-derived DIPG lines, and are reproducible across biological repeats, the fraction of cells in each cluster varies. This suggests that environmental conditions may affect the propensity of cells to be in each of the states. It is currently unclear how stable these epigenetic subpopulations are, and whether cells can transition from one state to the other. Follow-up studies in mice models as well as analysis of fresh tumors are needed to visualize these epigenetic states in-vivo, and further investigate whether they are correlated with distinct features in the tumor such as spatial localization of the cells, etc.

Our work establishes new modes for the analysis of CyTOF data, focusing on epigenetic markers that are expressed in gradients in all cells rather than proteins with binary expression patterns that are often analyzed by CyTOF (i.e., cell surface markers indicative of cell identity, etc.). We developed a normalization strategy to account for technical variation that likely stems from differences in permeability between cells during the staining protocol, and is thus highly important when measuring nuclear proteins. We then leveraged the single-cell data to extrapolate on crosstalk between epigenetic marks. Interestingly, we identified a high correlation between H4K16ac and $\mathrm{H} 3 \mathrm{~K} 4 \mathrm{me} 3$, which is supported by a reported interaction between the two writers that deposit these modifications: MOF and MLL1 ${ }^{47}$. The high correlation suggests the deposition of these two marks may be coordinated in diverse biological systems. Finally, we show that the multiple measurements from individual cells can be leveraged to identify epigenetic changes associated with distinct cell states, for example phases of the cell cycle or early versus late DNA synthesis. Using the core histones for normalization allowed us to follow the restoration of histone modifications 
bioRxiv preprint doi: https://doi.org/10.1101/2021.11.02.466907; this version posted November 4, 2021. The copyright holder for this preprint

(which was not certified by peer review) is the author/funder, who has granted bioRxiv a license to display the preprint in perpetuity. It is made available under aCC-BY-NC-ND 4.0 International license.

during S-phase, establishing the system as complementary to ChOR-seq ${ }^{53}$. This approach can be applied to address diverse biological questions related to cancer and cellular differentiation. 


\section{Methods}

\section{Cell culture}

HEK293 cells were grown in complete DMEM containing 10\% FBS (v/v) and 1\% P/S (v/v) on $10 \mathrm{~cm}$ dishes or 6-well plates and passaged by trypsination. So long as the cells were not induced with doxycycline, G418 antibiotic (Merck \#A1720) was added to the medium at every passage at $0.5 \mathrm{mg} / \mathrm{ml}$ to select against uninfected cells. After starting induction, the medium was replaced daily with fresh medium containing $1 \mu \mathrm{g} / \mathrm{ml}$ doxycycline (Merck \#D9891). Prior to CyTOF experiments, HEK293 cultures were washed once with PBS and thoroughly pipetted after trypsination to ensure single-cell separation. For immunofluorescence imaging, HEK293 cell were grown on glass coverslips (13 mm, No.1). DIPG cells were grown according to a previously published protocol $^{56}$ in T25 or T75 filter flasks (Thermo Fisher \#156367, 156499) in tumor stem medium (TSM) based on a 1:1 mixture of Neurobasal-A medium (Thermo Fisher \#10888022) and DMEM-F12 (Thermo Fisher \#11330032) and supplemented with: 10mM HEPES buffer (Thermo Fisher \#15630056), 1 mM MEM sodium-pyruvate (Thermo Fisher \#11360039), 0.1 mM MEM non-essential amino acids (Thermo Fisher \#11140035), 2 mM L-alanyl-L-glutamine dipeptide (Glutamax, Thermo Fisher \#35050038), antibiotic antimycotic (Thermo Fisher \#15240096), 2\% B27 supplement w/o vitamin A (v/v) (Thermo Fisher \#12587010), 20 ng/ml H-EGF (Shenandoah \#100-26), $20 \mathrm{ng} / \mathrm{ml}$ H-FGF-basic-154 (Shenandoah \#100-146), $10 \mathrm{ng} / \mathrm{ml}$ H-PDGF-AA (Shenandoah \#100-16), $10 \mathrm{ng} / \mathrm{ml}$ H-PDGF-BB (Shenandoah \#100-16) and $2 \mu \mathrm{g} / \mathrm{ml}$ heparin (Stem Cell Technologies \#07980). All cells were grown in a standard humidified cell culture incubator at $37{ }^{\circ} \mathrm{C}$ and $5 \% \mathrm{CO}_{2}$. Cell cultures were regularly tested for mycoplasma contamination.

\section{Antibody metal conjugation}

Antibodies were conjugated to metals using the Maxpar X8 Antibody Labeling Kit (Fluidigm) or the MIBItag Conjugation Kit (IONpath), following manufacturer protocols.

Metals for conjugation were chosen in a way that would minimize noise and spillover between channels. This was done according to guidelines appearing in Han et al. ${ }^{57}$. Tables S1 specifies the antibodies used and the metal assigned to each antibody.

\section{CyTOF sample preparation}

Sample preparation was similar to previously described protocols ${ }^{58}$. In short, cells were harvested into 5ml FACS tubes, washed with CyTOF PBS (Fluidigm \# 201058) then labeled with $1.25 \mu \mathrm{M}$ cisplatin (Fluidigm \#201064) for one minute to stain for dead cells. The cisplatin was then 
quenched with DMEM+10\% FBS. After washing with CyTOF staining buffer (Fluidigm kit \#201063), the cells were gently fixed with nuclear antigen staining working solution (Fluidigm kit \#201063) then permeabilized with CyTOF permeabilization buffer (Fluidigm kit \#201063) and barcoded with palladium barcodes (Fluidigm \#201060). The cells were then washed with CyTOF permeabilization buffer and the barcoded samples were combined before being incubated with antibodies in a solution containing 10\% NGS (Cell Signaling \#5425). Cells were then washed with CyTOF staining buffer and fixed with $10 \%$ formalin (Merck \#F5554) at $4{ }^{\circ} \mathrm{C}$ overnight with gentle rocking to prevent clumping. The formalin solution then had iridium (Fluidigm \#201192A) at a final concentration of $125 \mathrm{nM}$ added to it to label DNA. The cells were then washed with CyTOF staining buffer followed by CyTOF water (Fluidigm \#201069). The cells were then resuspended at a concentration of about $250 \mathrm{k} / \mathrm{ml}$ in CyTOF water containing 1:10 EQ Four Element Calibration Beads and filtered with a $35 \mu \mathrm{m}$ mesh before acquiring data on a Fluidigm Helios CyTOF device.

\section{CyTOF data analysis}

\section{i. Data processing}

CyTOF data underwent the following pre-processing prior to analysis: First, CyTOF software by Fluidigm was used to allow normalization and concatenation of the acquired data. Then, several gates were applied using the Cytobank platform: First, the normalization beads were gated out using the 140Ce channel. Then, single live cells were gated using the cisplatin 195Pt channel and the iridium DNA label in 193Ir channel. Further gates were applied using event parameters such as event length, width, center and residual, to keep only reliable events in the data, according to the guidelines outlined by Bagwell et $\mathrm{al}^{59}$. CyTOF software was then used for sample de-barcoding.

\section{ii. Data manipulation, transformation and scaling}

Before beginning the analysis procedure, the cells were gated using the core histones (H3, H3.3, and H4). The gate allowed only cells with a minimum dual count value of 5 for all core histones into the next steps of the analysis. The gating on the core histones was followed by a gate on a subset of the epigenetic markers (H3K36me3, H3K4me3, H3K36me2, H4K16ac, H2Aub, H3K4me1, H3K64ac, H3K27ac, H3K9ac, H3K27me3, and H3K9me3), gating for a minimum raw value of 2 for said modifications. To deal with the different sensitivity of the CyTOF apparatus to the various markers a hyperbolic arcsine transform (with a scale factor of 5) was first applied to the data, followed by a Z-transform:

$$
M^{i, j}=\operatorname{arcsinh} \frac{M^{i, j}}{5}
$$




$$
M^{i, j}=\frac{M^{i, j}-\mu^{j}}{\sigma^{j}}
$$

Where $\mathrm{i}$ denotes the observation, $\mathrm{j}$ denotes the column (modification), $\mu^{j}$ is the mean of column $\mathrm{j}$, and $\sigma^{j}$ is its standard deviation.

Z-transform scaling was done at the same time on samples that were acquired together, such as the HEK293 WT and H3-K27M cell lines.

\section{iii. Epigenetic markers normalization}

Based on the assumption that the core histones $\mathrm{H} 3, \mathrm{H} 3.3$, and $\mathrm{H} 4$ should be expressed at a similar level in all cells, systematic effects were removed from the measurement procedure by subtracting a linear combination of the three core histones from the epigenetic values of all observations:

$$
M^{i, j}=M^{i, j}-\alpha H_{3.3}^{i}-\beta H_{4}^{j}-\gamma H_{3}^{j}
$$

where the coefficients $\alpha, \beta$, and $\gamma$ are chosen by a least squares minimization procedure to minimize the sum of the variances of the core histones across all observations of a single sample: $\sigma\left(H_{3.3}\right)+$ $\sigma\left(H_{4}\right)+\sigma\left(H_{3}\right)$. Normalization was done separately for each sample. A reduction of a few tens of percent in the sum of variances was observed after the subtraction, indicating the removal of systematic effects.

\section{Western blot analysis}

For western blots analysis, cell pellets were resuspended in Laemmli sample buffer (Bio-Rad \#1610747) containing 50mM DTT and vortexed and heated up to $98^{\circ} \mathrm{C}$ three times. The prepared pellets were loaded into a TG gels (Thermo Fisher \#XP04205). Electrophoresis was carried out in TG-SDS buffer for 1-1.5 hours at 100V. After electrophoresis, the proteins were transferred onto nitrocellulose membranes using a commercial transfer kit (Bio-Rad \#1704158). After transfer, the membranes were briefly rinsed in TBS then blocked with 5\% milk powder in TBST for 45 minutes $(\mathrm{w} / \mathrm{v})$. The membranes were then rinsed three times in TBST and incubated with primary antibodies overnight at $4{ }^{\circ} \mathrm{C}$ with gentle rocking. Primary antibodies (Table S2) were diluted according to manufacturer instructions in TBST containing 5\% BSA (w/v) and $0.04 \%$ sodium-azide $(\mathrm{w} / \mathrm{v})$. The following day the membranes were rinsed three times in TBST then incubated with anti-mouse or anti-rabbit HRP-conjugated secondary antibodies diluted according to manufacturer instructions in a solution containing $5 \%$ milk powder in TBST $(\mathrm{w} / \mathrm{v})$. The membranes were then rinsed three times 
with TBST and dunked in an ECL mixture (Bio-Rad \#1705061) prior to exposure. Membranes were imaged using a Bio-Rad ChemiDoc MP imaging system.

\section{Immunofluorescence imaging and analysis}

Once ready for imaging, cells were fixed on coverslips with a $4 \%$ formaldehyde solution in PBS for 20 minutes. The formaldehyde was then removed, and the coverslips were rinsed three times with PBS. Cells were then permeabilized with a $0.1 \%$ triton-X100 solution in PBS for 20 minutes. The triton solution was then removed, and the coverslips were rinsed three times with PBS. The coverslips were then blocked with a $10 \%$ BSA solution in PBS at $4{ }^{\circ} \mathrm{C}$ overnight. The following day the coverslips were stained with primary antibodies (Table S2) diluted according to manufacturer instructions in a 1\% BSA solution in PBS and incubated for 90 minutes on the benchtop. The coverslips were then washed twice in PBS and incubated for 30 minutes with secondary antibodies. After said incubation the coverslips were washed twice with PBS and stained with DAPI working solution for 5 minutes. After DAPI staining the coverslips were washed once with PBS and mounted on $25 \times 75 \times 1 \mathrm{~mm}$ glass slides with ProLong Gold Antifade Mountant (Thermo Fisher P10144). The slides were visualized on a Nikon Eclipse Ti2 microscope with a Yokogawa CSU-W1 confocal scanner unit and photographed using a Photometrics Prime 95B CMOS camera.

Nuclear area was measured manually using Fiji based on ImageJ version $1.53 \mathrm{c}$ available on imagej.net

\section{Acknowledgments}

We thank Y. Aylon and I. Tirosh for their important and helpful input. We are grateful for M. Monje and N.Jabado for generously sharing with us the SU-DIPG48, SU-DIPG6, SU-DIPG13 ${ }^{56}$ and the isogenic SU-DIPG13 H3-K27M / KO cultures ${ }^{32}$. Figure 1A was created using biorender.com. Funding: E.S. is an incumbent of the Lisa and Jeffrey Aronin Family Career Development chair. This research was supported by grants from the European Research Council (ERC801655), The Israeli Science Foundation (1881/19), The German-Israeli Foundation for Scientific Research and Development and Minerva. 


\section{Author Contributions}

N.H, T.M, G.R and E.S designed the study, contributed conceptually to the data analysis and wrote the manuscript. N.H, T.M and O.B established the antibodies panel and conducted the experiments. G.R and N.H performed CyTOF data analysis. T.S assisted in performing the CyTOF runs as well as designing the panel. N.F. and O.B. generated the HEK293 inducible system.

\section{Competing Interests statement}

The authors declare no competing interests.

\section{References:}

1. WA, F., E, G. \& BE, B. Epigenetic plasticity and the hallmarks of cancer. Science 357, (2017).

2. AM, V.\& C, K. Chromatin regulatory mechanisms and therapeutic opportunities in cancer. Nat. Cell Biol. 21, 152-161 (2019).

3. Z, Z. \& A, S. Epigenetic modifications of histones in cancer. Genome Biol. 20, (2019).

4. T, B. \& J, H. Unravelling biology and shifting paradigms in cancer with single-cell sequencing. Nat. Rev. Cancer 17, 557-569 (2017).

5. ML, S. \& I, T. Single-Cell RNA Sequencing in Cancer: Lessons Learned and Emerging Challenges. Mol. Cell 75, 7-12 (2019).

6. Shema, E., Bernstein, B. E. \& Buenrostro, J. D. Single-cell and single-molecule epigenomics to uncover genome regulation at unprecedented resolution. Nature Genetics 51, 19-25 (2019).

7. Smallwood, S. A. et al. Single-cell genome-wide bisulfite sequencing for assessing epigenetic heterogeneity. Nat. Methods 11, 817-820 (2014).

8. Cusanovich, D. A. et al. Multiplex single-cell profiling of chromatin accessibility by combinatorial cellular indexing. Science (80-. ). 348, 910-914 (2015). 
9. C, L., P, H. \& JR, E. Dynamic DNA methylation: In the right place at the right time. Science 361, 1336-1340 (2018).

10. G, K., O, S. \& W, R. Single-cell epigenomics: Recording the past and predicting the future. Science 358, 69-75 (2017).

11. Bartosovic, M., Kabbe, M. \& Castelo-Branco, G. Single-cell CUT\&Tag profiles histone modifications and transcription factors in complex tissues. Nat. Biotechnol. (2021). doi:10.1038/s41587-021-00869-9

12. Wu, S. J. et al. Single-cell CUT\&Tag analysis of chromatin modifications in differentiation and tumor progression. Nat. Biotechnol. (2021). doi:10.1038/s41587-021$00865-\mathrm{z}$

13. Rotem, A. et al. Single-cell ChIP-seq reveals cell subpopulations defined by chromatin state. Nat. Biotechnol. 33, 1165-1172 (2015).

14. Grosselin, K. et al. High-throughput single-cell ChIP-seq identifies heterogeneity of chromatin states in breast cancer. Nat. Genet. 51, 1060-1066 (2019).

15. Boettiger, A. N. et al. Super-resolution imaging reveals distinct chromatin folding for different epigenetic states. Nature 529, 418-422 (2016).

16. Xu, J. et al. Super-Resolution Imaging of Higher-Order Chromatin Structures at Different Epigenomic States in Single Mammalian Cells. Cell Rep. 24, 873-882 (2018).

17. Woodworth, M. A. et al. Multiplexed single-cell profiling of chromatin states at genomic loci by expansion microscopy Marcus. Nucleic Acids Res. 1-15 (2021). doi:10.1093/nar/gkab423

18. Cheung, P. et al. Single-Cell Chromatin Modification Profiling Reveals Increased Epigenetic Variations with Aging. Cell 173, 1385-1397.e14 (2018).

19. Wimmers, F. et al. Single-cell analysis of the epigenomic and transcriptional landscape of innate immunity to seasonal and adjuvanted pandemic influenza vaccination in humans. medRxiv 2021.05.24.21253087 (2021). doi:10.1101/2021.05.24.21253087

20. Wu, G. et al. Somatic histone $\mathrm{H} 3$ alterations in pediatric diffuse intrinsic pontine gliomas and non-brainstem glioblastomas. Nat. Genet. 44, 251-253 (2012).

21. Schwartzentruber, J. et al. Driver mutations in histone H3.3 and chromatin remodelling 
genes in paediatric glioblastoma. Nature 482, 226-231 (2012).

22. Phillips, R. E., Soshnev, A. A. \& Allis, C. D. Epigenomic Reprogramming as a Driver of Malignant Glioma. Cancer Cell 38, 647-660 (2020).

23. BA, N. et al. The expanding landscape of 'oncohistone' mutations in human cancers. Nature 567, 473-478 (2019).

24. M, F. \& M, M. Developmental origins and emerging therapeutic opportunities for childhood cancer. Nat. Med. 25, 367-376 (2019).

25. Zhang, X. \& Zhang, Z. Oncohistone Mutations in Diffuse Intrinsic Pontine Glioma. Trends in Cancer 5, 799-808 (2019).

26. Bender, S. et al. Reduced H3K27me3 and DNA Hypomethylation Are Major Drivers of Gene Expression in K27M Mutant Pediatric High-Grade Gliomas. Cancer Cell 24, 660 672 (2013).

27. Lewis, P. W. et al. Inhibition of PRC2 activity by a gain-of-function $\mathrm{H} 3$ mutation found in pediatric glioblastoma. Science (80-. ). 340, 857-861 (2013).

28. Chan, K. M. et al. The histone H3.3K27M mutation in pediatric glioma reprograms H3K27 methylation and gene expression. Genes Dev. 27, 985-990 (2013).

29. Piunti, A. et al. Therapeutic targeting of polycomb and BET bromodomain proteins in diffuse intrinsic pontine gliomas. Nat. Med. 23, 493-500 (2017).

30. B, K., AS, H., S, D. \& N, J. Polycomb repressive complex 2 in the driver's seat of childhood and young adult brain tumours. Trends Cell Biol. (2021).

doi:10.1016/J.TCB.2021.05.006

31. McInnes, L., Healy, J., Saul, N. \& Großberger, L. UMAP: Uniform Manifold Approximation and Projection. J. Open Source Softw. 3, 861 (2018).

32. Harutyunyan, A. S. et al. H3K27M in Gliomas Causes a One-Step Decrease in H3K27 Methylation and Reduced Spreading within the Constraints of H3K36 Methylation. Cell Rep. 33, (2020).

33. EM, D. et al. Cathepsin L proteolytically processes histone $\mathrm{H} 3$ during mouse embryonic stem cell differentiation. Cell 135, 284-294 (2008).

34. K, K. et al. MMP-9 facilitates selective proteolysis of the histone $\mathrm{H} 3$ tail at genes 
necessary for proficient osteoclastogenesis. Genes Dev. 30, 208-219 (2016).

35. Zhou, P., Wu, E., Alam, H. B. \& Li, Y. Histone Cleavage as a Mechanism for Epigenetic Regulation: Current Insights and Perspectives. Curr. Mol. Med. 14, 1164 (2014).

36. Chen, T. \& Guestrin, C. XGBoost: A Scalable Tree Boosting System. in Proceedings of the 22nd ACM SIGKDD International Conference on Knowledge Discovery and Data Mining (ACM).

37. Rice, J. C. \& Allis, C. D. Histone methylation versus histone acetylation: New insights into epigenetic regulation. Current Opinion in Cell Biology 13, 263-273 (2001).

38. Alabert, C. et al. Two distinct modes for propagation of histone PTMs across the cell cycle. Genes Dev. 29, 585-590 (2015).

39. Torres, C. M. et al. The linker histone H1.0 generates epigenetic and functional intratumor heterogeneity. Science (80-. ). 353, (2016).

40. Suvà, M. L. et al. Reconstructing and reprogramming the tumor-propagating potential of glioblastoma stem-like cells. Cell 157, 580-594 (2014).

41. Pajovic, S. et al. Epigenetic activation of a RAS/MYC axis in H3.3K27M-driven cancer. Nat. Commun. 11, (2020).

42. Neftel, C. et al. An Integrative Model of Cellular States, Plasticity, and Genetics for Glioblastoma. Cell 178, 835-849.e21 (2019).

43. Filbin, M. G. et al. Developmental and oncogenic programs in H3K27M gliomas dissected by single-cell RNA-seq.

44. Di Cerbo, V. et al. Acetylation of histone $\mathrm{H} 3$ at lysine 64 regulates nucleosome dynamics and facilitates transcription. Elife 2014, 1632 (2014).

45. Zhou, W. et al. Acetylation of H3K4, H3K9, and H3K27 mediated by p300 regulates the expression of GATA4 in cardiocytes. Genes Dis. 6, 318-325 (2019).

46. Smith, E. R. et al. A Human Protein Complex Homologous to the Drosophila MSL Complex Is Responsible for the Majority of Histone H4 Acetylation at Lysine 16. Mol. Cell. Biol. 26, 387-387 (2006).

47. Dou, Y. et al. Physical association and coordinate function of the H3 K4 methyltransferase MLL1 and the H4 K16 acetyltransferase MOF. Cell 121, 873-885 
(2005).

48. Zhang, Y. et al. Selective binding of the PHD6 finger of MLL4 to histone H4K16ac links MLL4 and MOF. Nat. Commun. 10, (2019).

49. Yu, J. R., Lee, C. H., Oksuz, O., Stafford, J. M. \& Reinberg, D. PRC2 is high maintenance. Genes and Development 33, 903-935 (2019).

50. Ernst, J. et al. Mapping and analysis of chromatin state dynamics in nine human cell types. Nature 473, 43-49 (2011).

51. Couture, J. F., Collazo, E., Ortiz-Tello, P. A., Brunzelle, J. S. \& Trievel, R. C. Specificity and mechanism of JMJD2A, a trimethyllysine-specific histone demethylase. Nat. Struct. Mol. Biol. 14, 689-695 (2007).

52. Kheir, T. B. \& Lund, A. H. Epigenetic dynamics across the cell cycle. Essays Biochem. 48, 107-120 (2010).

53. Reverón-Gómez, N. et al. Accurate Recycling of Parental Histones Reproduces the Histone Modification Landscape during DNA Replication. Mol. Cell 72, 239-249.e5 (2018).

54. Klein, K. N. et al. Replication timing maintains the global epigenetic state in human cells. Science (80-. ). 372, 371-378 (2021).

55. Schachner, L. F. et al. Decoding the protein composition of whole nucleosomes with NucMS. Nat. Methods 18, 303-308 (2021).

56. Grasso, C. S. et al. Functionally defined therapeutic targets in diffuse intrinsic pontine glioma. Nat. Med. 21, 555-559 (2015).

57. Han, G., Spitzer, M. H., Bendall, S. C., Fantl, W. J. \& Nolan, G. P. Metal-isotope-tagged monoclonal antibodies for high-dimensional mass cytometry. Nat. Protoc. 20181310 13, 2121-2148 (2018).

58. Palii, C. G. et al. Single-Cell Proteomics Reveal that Quantitative Changes in Coexpressed Lineage-Specific Transcription Factors Determine Cell Fate. Cell Stem Cell 24, 812 (2019).

59. Bagwell, C. B. et al. Automated Data Cleanup for Mass Cytometry. Cytom. Part A 97, 184-198 (2020). 
bioRxiv preprint doi: https://doi.org/10.1101/2021.11.02.466907; this version posted November 4, 2021. The copyright holder for this preprint

(which was not certified by peer review) is the author/funder, who has granted bioRxiv a license to display the preprint in perpetuity. It is made available under aCC-BY-NC-ND 4.0 International license.

60. Sharko, J., Grinstein, G. \& Marx, K. A. Vectorized radviz and its application to multiple cluster datasets. in IEEE Transactions on Visualization and Computer Graphics 14, 1444 1451 (2008). 


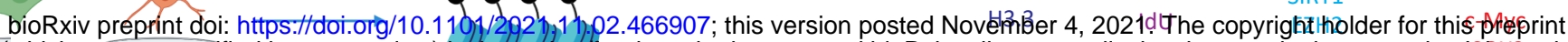
(which was not certified by peer review) is the authorlfunder, who has granted bioRxiv a lidense to display the preprint in perpetuity. Ifis

H3-K27M Systems

\section{CyTOF}

Single-Cell Epigenetic Analysis
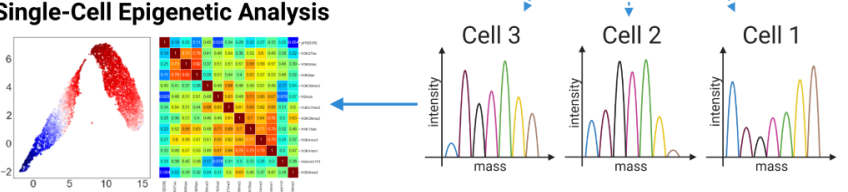

B
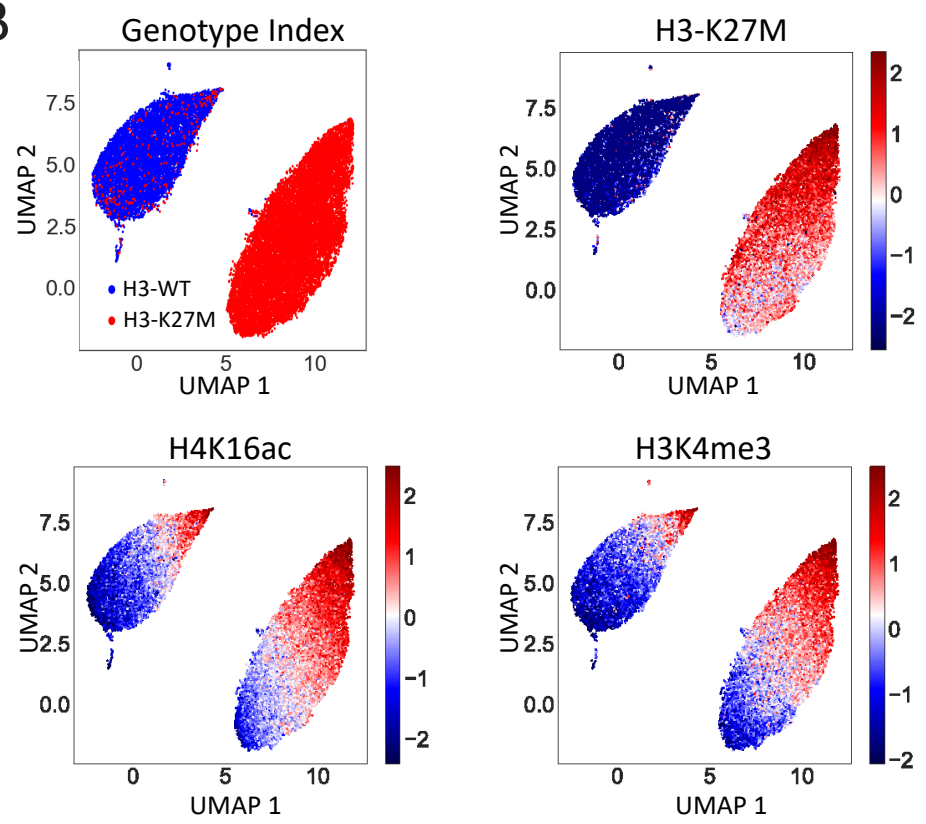

C

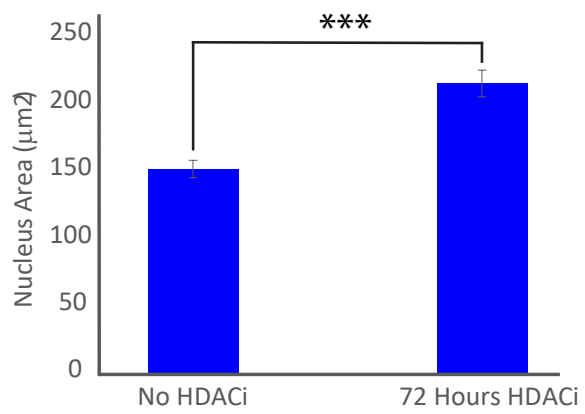

Histone Modifications

Glioma Lineage

$\begin{array}{ccccc}\text { H3K27me3 } & \text { H3K36me3 } & \text { H3K4me1 } & \text { yH2A.X } & \text { CXCR4 } \\ \text { H3K27ac } & \text { H3K36me2 } & \text { H3K79me2 } & \text { H2AK119ub } & \text { GFAP } \\ \text { H3K9me3 } & \text { H3K9ac } & \text { H4K20me3 } & \text { Cleaved H3 } & \text { DLL3 } \\ \text { H3K4me3 } & \text { H4K16ac } & \text { H3K64ac } & & \text { MBP }\end{array}$
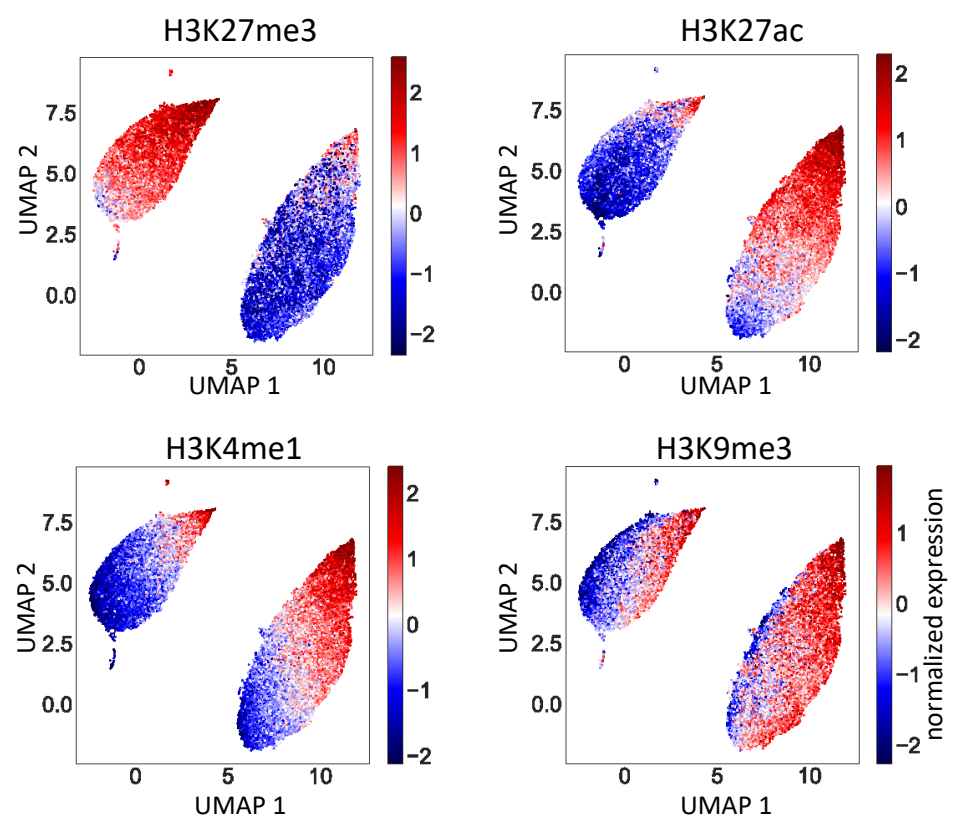

D

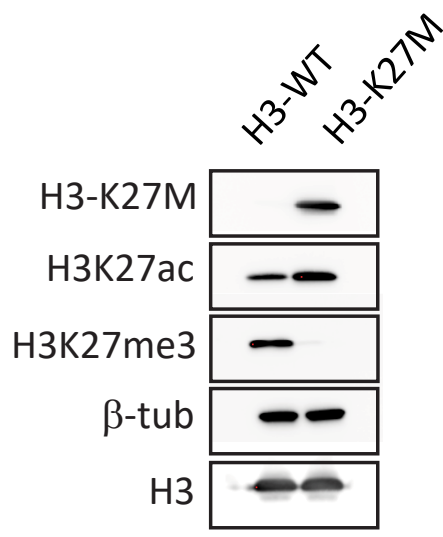

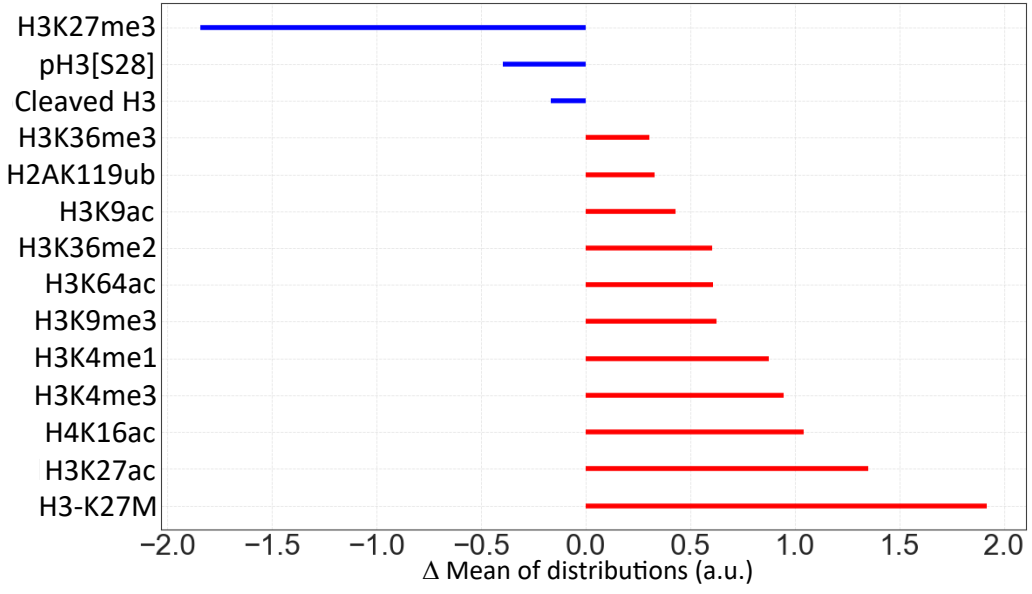

E

G

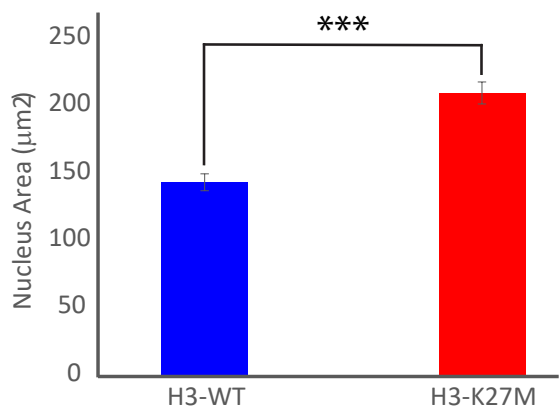

$E$

F
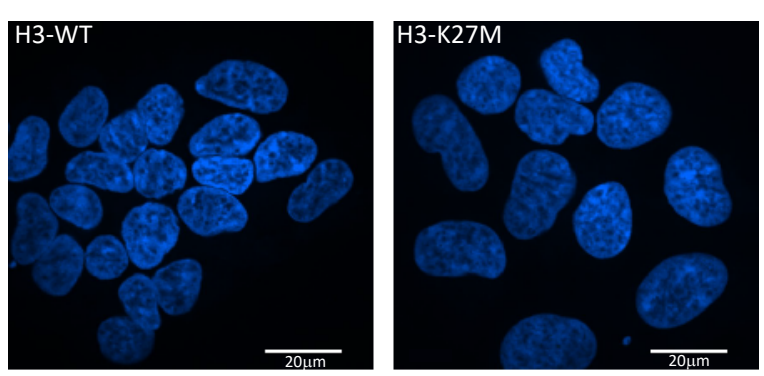

$\mathrm{H}$

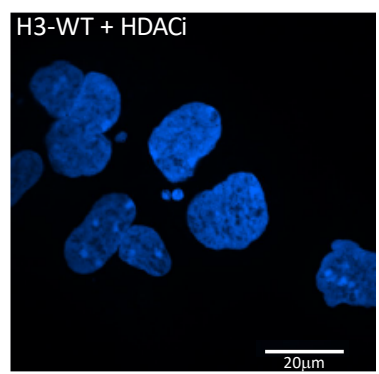




\section{Figure 1: High-dimensional single-cell analysis of H3-K27M-induced epigenetic alterations}

(A) Left: Scheme of CyTOF experimental setup and analysis for H3-K27M models. Right: Epigenetic-oriented antibody panel. (B-C) HEK293 cells were induced for 10 days to express H3WT or H3-K27M, and analyzed by CyTOF. (B) UMAP of CyTOF measurements after scaling and normalization (see Methods). The H3-K27M signal was not included in the UMAP algorithm. Isogenic cells expressing H3-K27M are clustered separately from WT cells. (C) Fold change differences between the WT and H3-K27M expressing cells for the indicated epigenetic modifications. Mean values (after transformation, scaling and normalization) of the WT were subtracted from the mutant. (D) Western blot analysis of HEK293 cells induced as in b-c with the indicated antibodies. H3-K27M is robustly induced, leading to reduced levels of H3K27me3 concomitant with an increase in $\mathrm{H} 3 \mathrm{~K} 27 \mathrm{ac}$. Beta tubulin and $\mathrm{H} 3$ represent loading controls. (E-F) HEK293 cells were induced to express WT or H3-K27M for 72 hours, followed by DAPI staining to label nuclei. (E) Quantification of nuclei size ( $\mathrm{n}=90$, p-value $=3.2 \mathrm{e}-10)$, one-sided Student's ttest was performed. Error bars represent SE. (F) Representative confocal images (scale $=20 \mu \mathrm{m}$ ). (G-H) HEK293 cells were treated with the HDAC inhibitor (HDACi) Vorinostat for the indicated times, and stained with DAPI. (G) Nuclei size was calculated as in e $(n=90, p$-value $=1.3 \mathrm{e}-7)$. $(\mathbf{H})$ Representative confocal images (scale $=20 \mu \mathrm{m}$ ). Cells expressing H3-K27M or treated with HDACi have enlarged nuclei. 


\section{Figure 2: Two distinct epigenetic populations identified in H3-K27M tumor-derived cells}

(A) SU-DIPG48 tumor-derived cells expressing WT H3 (blue) and SU-DIPG13 expressing H3K27M (red) were analyzed by CyTOF. UMAP based on all epigenetic modifications measured shows separate clustering of the two tumor-derived lines (red/blue indicates the sample index/barcode). Also shown are the scaled, normalized levels of H3-K27M, H3K27ac and H3K27me3 in these cells. (B) UMAP of SU-DIPG13 cells based on epigenetic modifications as in a, showing two distinct clusters. Levels of the indicated modifications in these two clusters are shown. (C) Spring plot $^{60}$ graphical representation of the gradient boosting algorithm, demonstrating the contribution of the top seven influencing epigenetic markers in the allocation of cells to clusters. (D) Fold change differences between the two clusters identified in SU-DIPG13 for the indicated epigenetic modifications. Mean values (after transformation, scaling and normalization) of the H3K27M-low cluster were subtracted from the H3-K27M-high cluster. 
Fig. 3

A HEK293

B

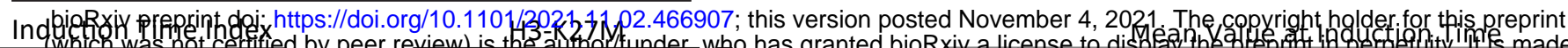

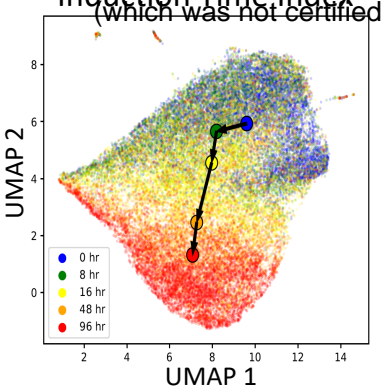

H3K27me3
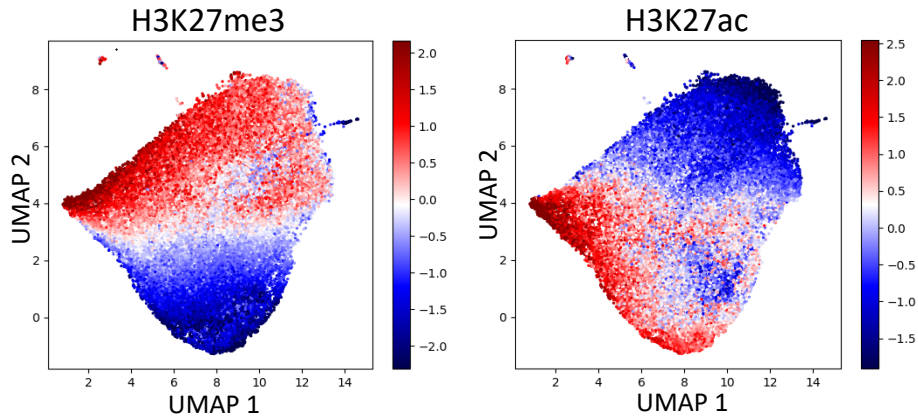

D

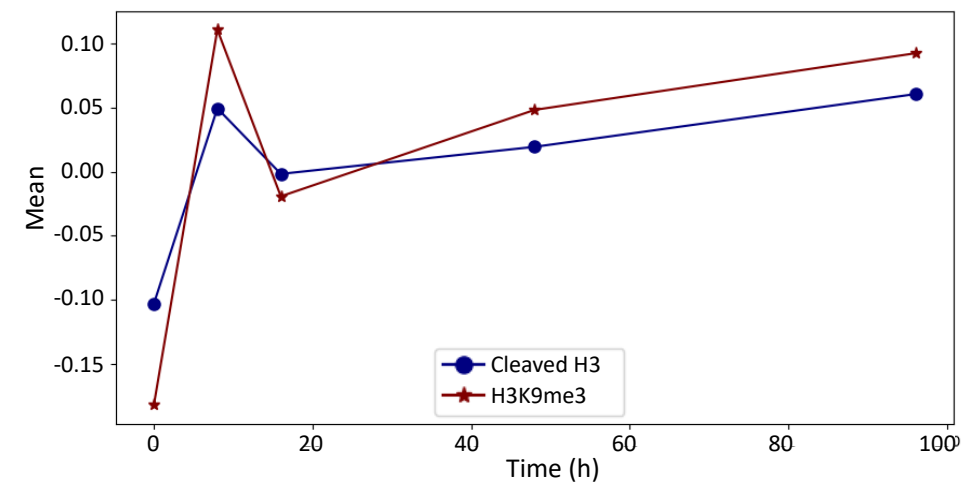




\section{Figure 3: Dynamics of H3-K27M-induced epigenetic alterations}

(A) HEK293 cells were induced to express H3-K27M for 8, 16, 48 and 96 hours, compared to cells expressing WT-H3 (marked as zero induction of H3-K27M), and analyzed by CyTOF with a panel of six epigenetic modifications, H3-K27M, and the core histones for normalization. UMAP based on all epigenetic modifications measured shows spatial continuous separation of the cells by their time of induction. Also shown are the scaled, normalized levels of H3-K27M, H3K27ac and H3K27me3. (B) The mean of distribution of the indicated modifications at the different time points. Dot size represents the sample variance. $(\mathbf{C}, \mathbf{D})$ Mean values of the indicated modifications as a function of $\mathrm{H} 3-\mathrm{K} 27 \mathrm{M}$ induction time. While the modifications in (C) show gradual increase/decrease with H3-K27M expression, cleaved H3 and H3K9me3 (D) are distinctly affected by differences in $\mathrm{H} 3-\mathrm{K} 27 \mathrm{M}$ levels. 
Fig. 4

A

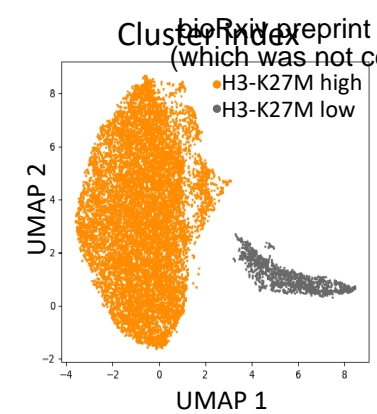

C

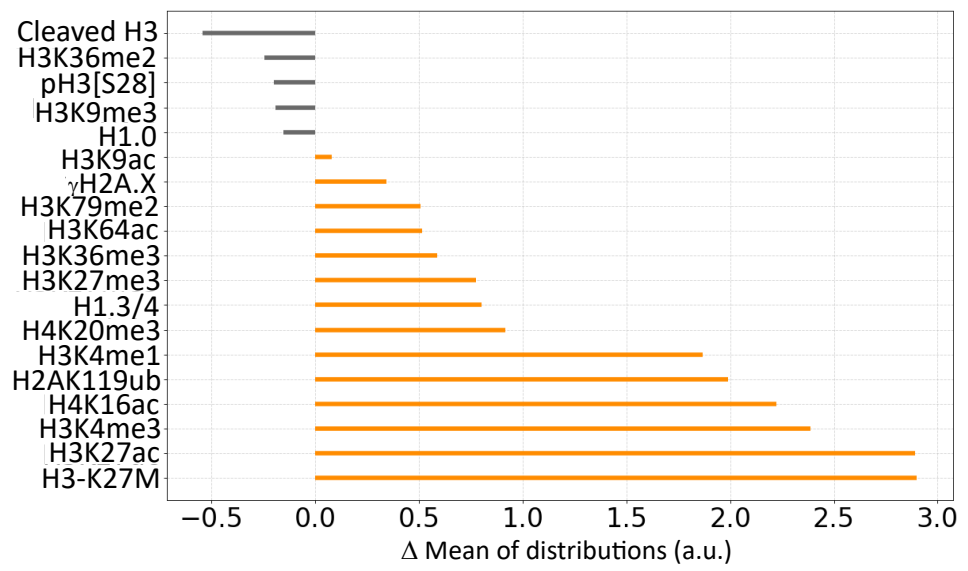

$\mathrm{F}$
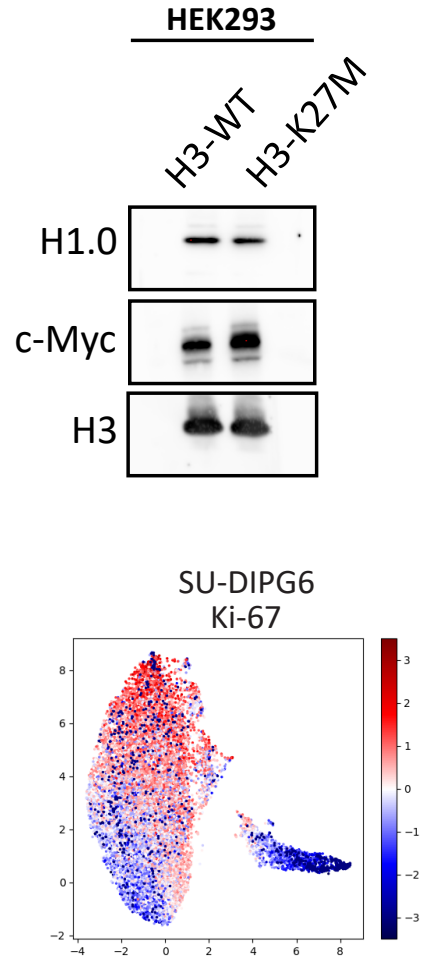

K
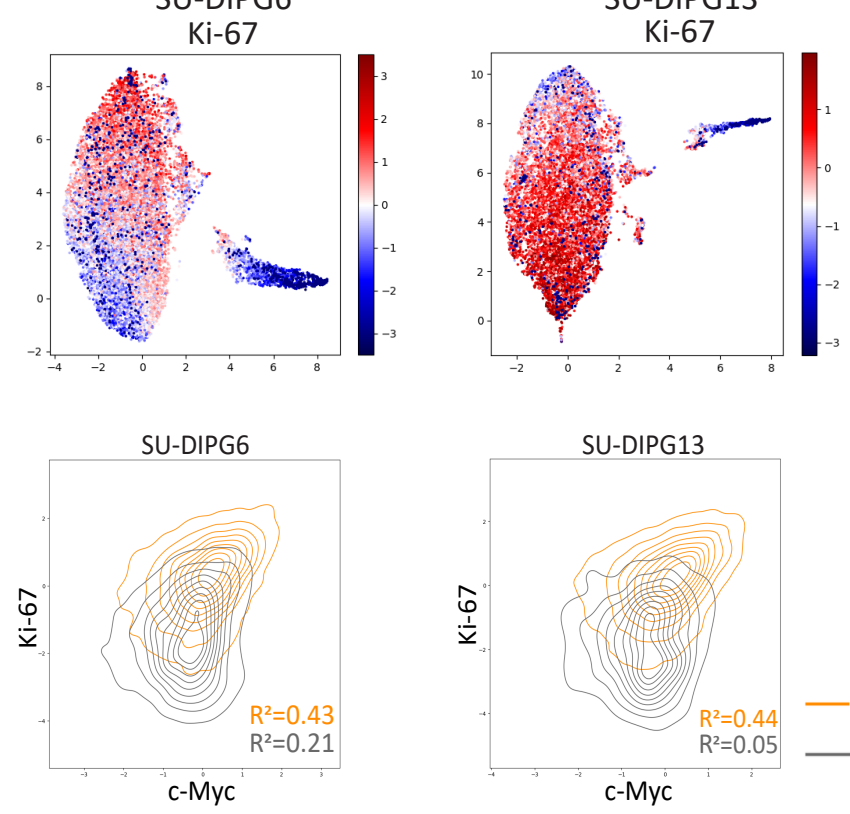

G
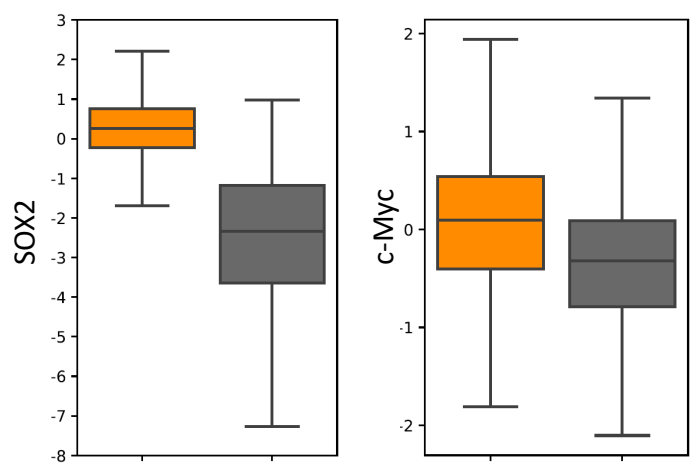

SU-DIPG13

SU-DIPG13

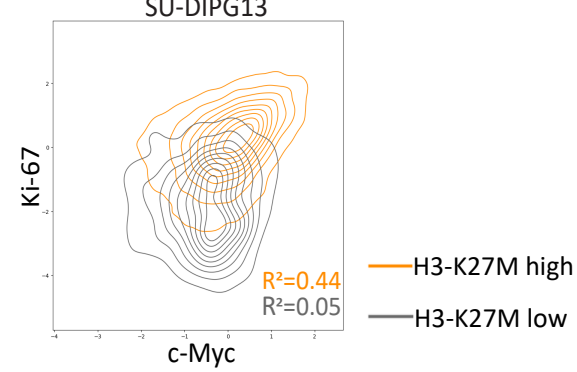

$\mathrm{H}$

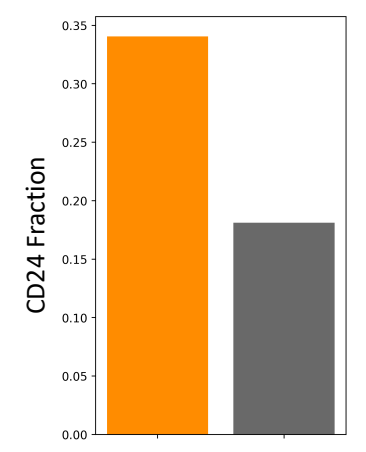

J

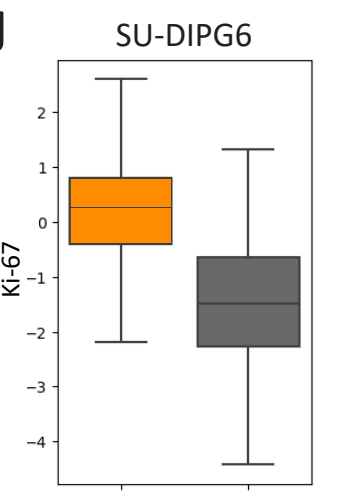

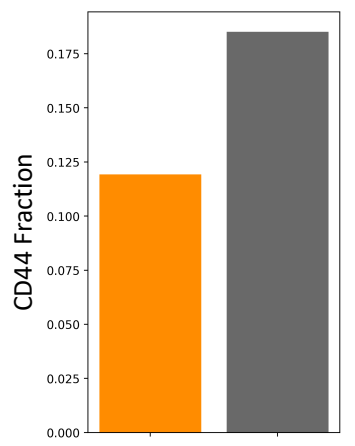

E
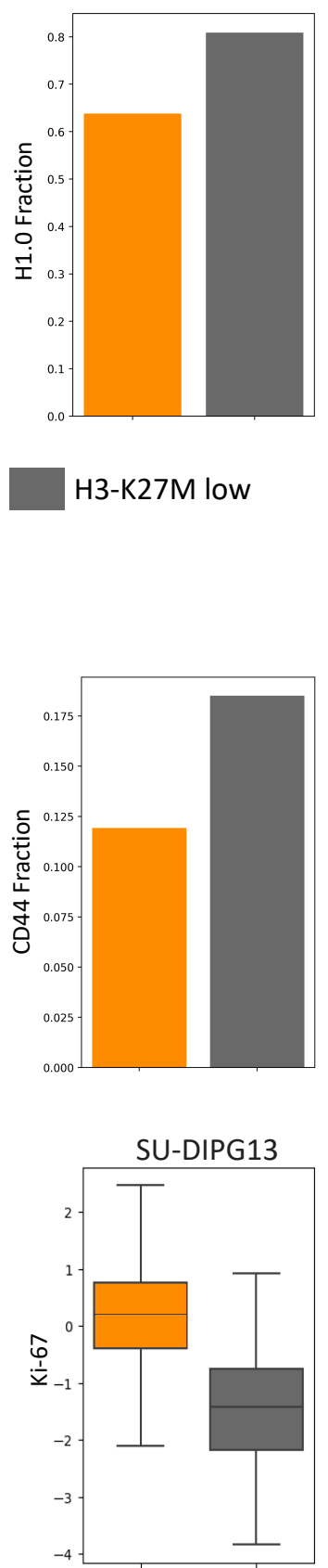
Figure 4: The two epigenetic states are robust across patients and show distinct proliferation capacity and expression of oncogenic and cancer stem-cells markers

(A) UMAP of SU-DIPG6 cells based on all the epigenetic modifications measured, showing two distinct clusters. Levels of H3-K27M, the histone variant H1.0, and the transcription factor SOX2 in the two clusters are shown. (B) UMAP of SU-DIPG13 (green) and SU-DIPG6 (purple) analyzed simultaneously in the CyTOF. Colors represent sample's barcode, indicating that the two lines, derived from different patients, are interchangeable and that both show the two epigenetic clusters. (C) Fold change differences between the two clusters identified in SU-DIPG6 for the indicated epigenetic modifications. Mean values (after transformation, scaling and normalization) of the H3K27M-low cluster were subtracted from the H3-K27M-high cluster. (D) Box plots depicting CyTOF measurements of EZH2 (transformed and scaled) in SU-DIPG6 H3-K27M-high and H3K27M-low clusters. (E) Fraction of cells positive for H1.0 in the two clusters. (F) Western blot analysis of HEK293 cells induced for 10 days to express H3-WT or H3-K27M. H3-K27M expression leads to downregulation of H1.0 and upregulation of c-Myc. H3 represents loading control. (G) Box plots depicting CyTOF measurements of SOX2 and c-Myc (transformed and scaled) in SU-DIPG6 H3-K27M-high and H3-K27M-low clusters. (H) Fraction of cells positive for CD24 and CD44 in the two clusters. (I) UMAP of SU-DIPG6 and SU-DIPG13 cells as in a. Shown are the scaled expression levels of the proliferation marker Ki-67 in the two epigenetic clusters. H3-K27M-high cluster is associated with increased Ki-67, indicating of higher proliferation. (J) Box plots of Ki-67 expression levels in the two clusters. (K) Contour plots of cMyc versus Ki-67 in SU-DIPG6 and SU-DUPG13 H3-K27M-high (orange) and H3-K27M-low (gray) clusters. 
Fig. 5

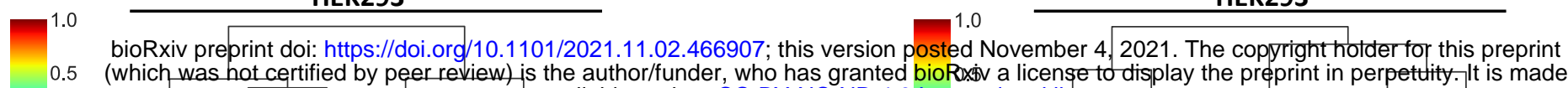

0

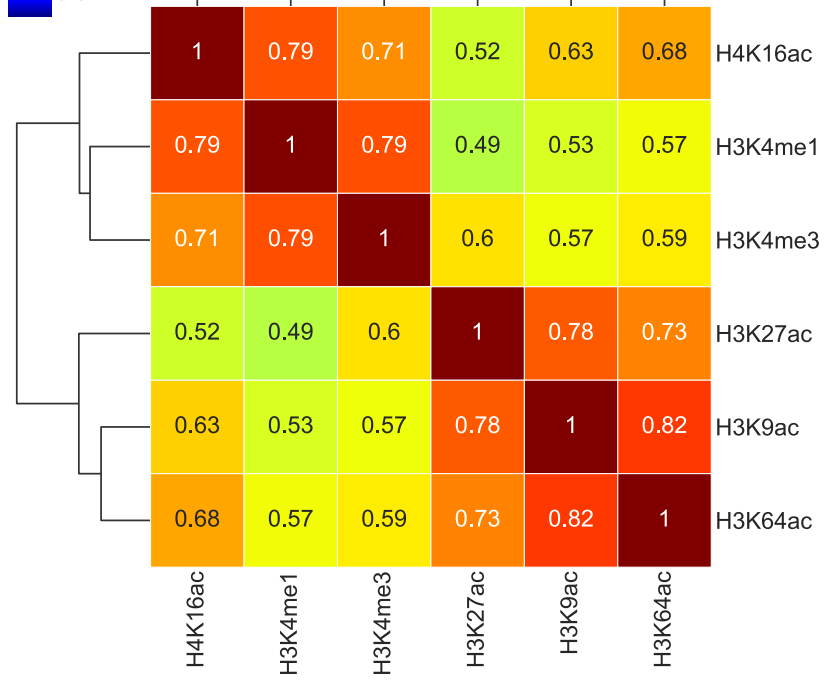

C
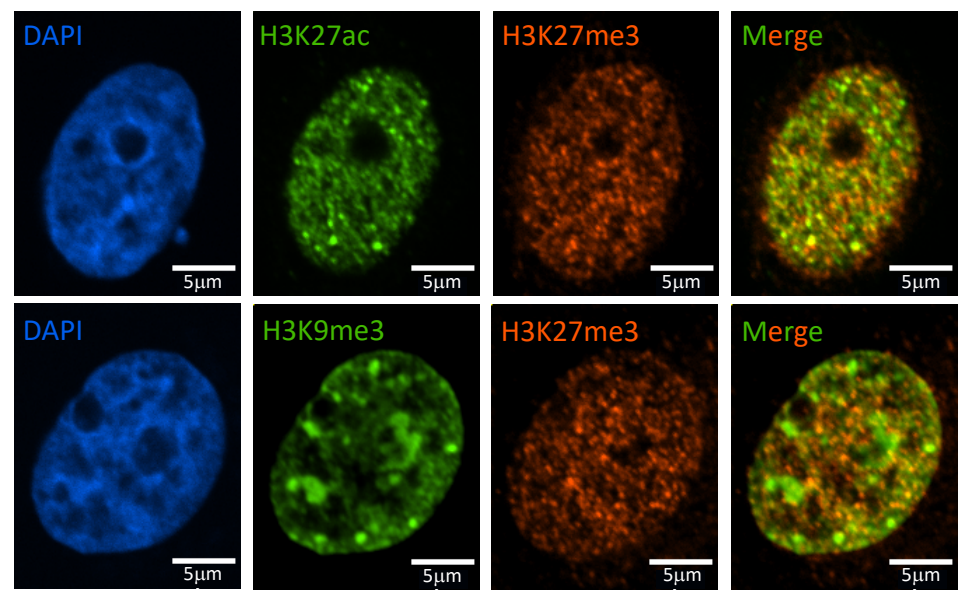

E

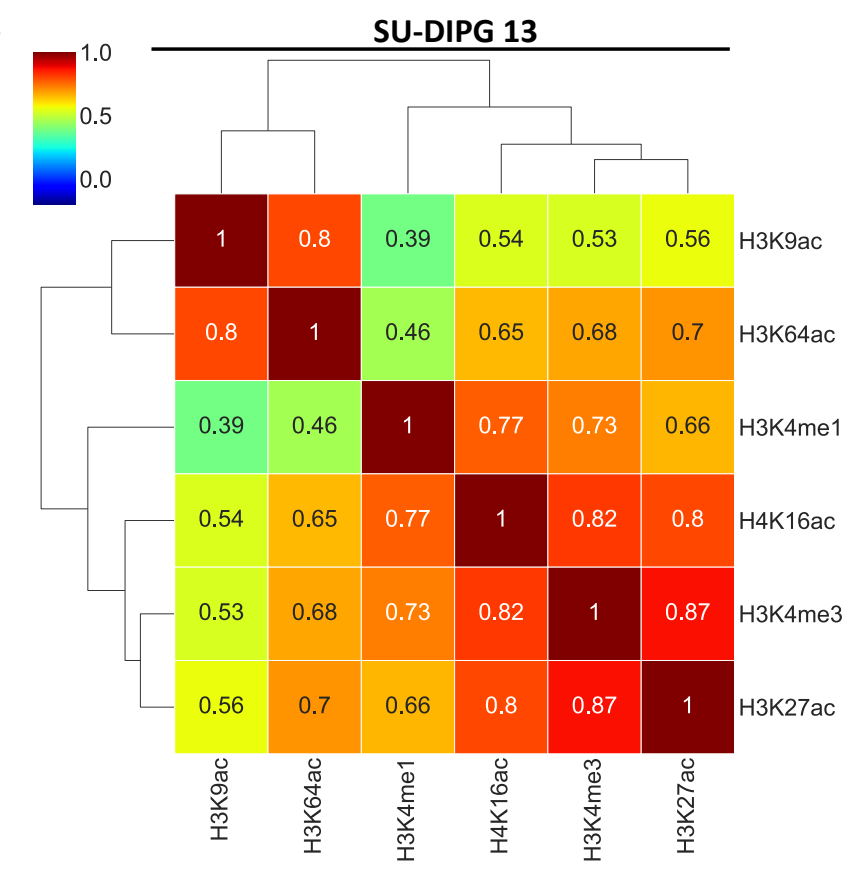

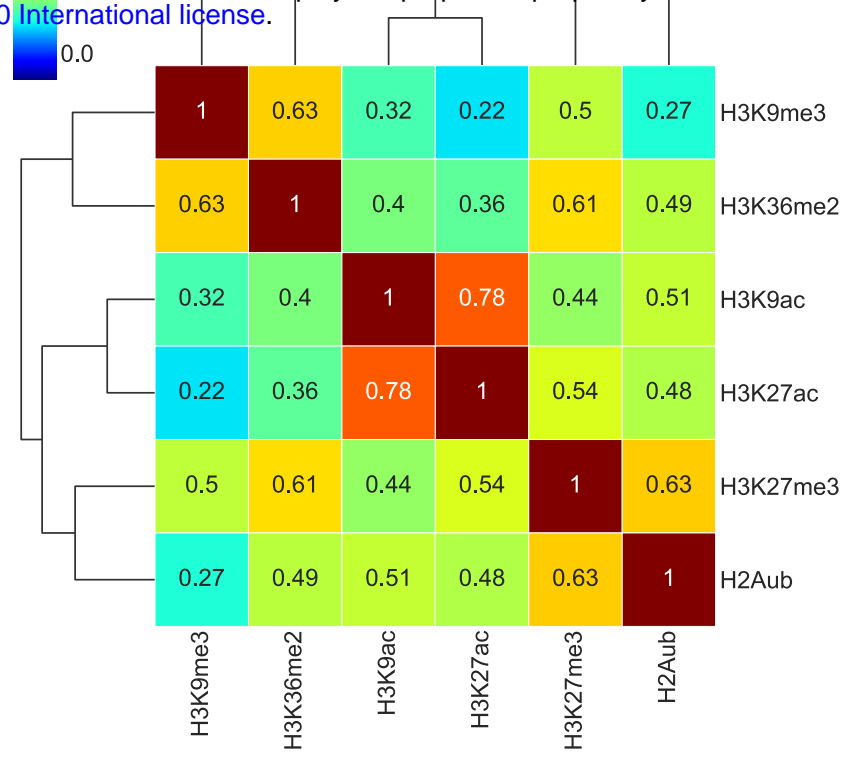

D

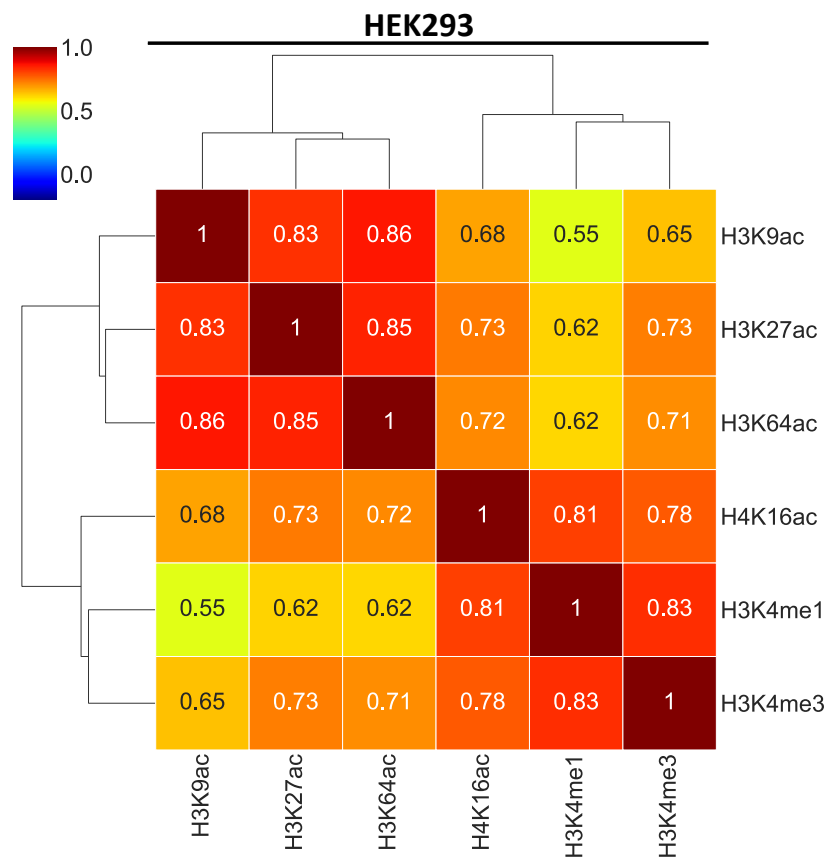




\section{Figure 5: Single cell pairwise correlations suggest co-regulation between epigenetic marks}

(A-B) Pearson pairwise correlation between histone modifications measured by CyTOF (after transformation, scaling and normalization) in WT HEK293 cells. (A) Coefficient of determination $\left(\mathrm{R}^{2}\right)$ heat map matrix of active histone modifications. The highest correlations are observed between $\mathrm{H} 3$ acetylations on lysine residues 9,27 and 64. H4 acetylation on lysine 16 is less correlated with H3 acetylations, and instead shows a high correlation with H3K4me1/3. (B) Coefficient of determination $\left(\mathrm{R}^{2}\right)$ heat map matrix of repressive histone modifications. H3K27me3 is highly correlated with $\mathrm{H} 2 \mathrm{Aub}$, but also shows relatively high correlation with the open chromatin marks H3K27ac and H3K9ac. (C) Immunofluorescence staining of MCF7 cells with the indicated antibodies. DAPI was used for DNA labeling. H3K9me3 is localized to discrete foci in the nuclei, as opposed to $\mathrm{H} 3 \mathrm{~K} 27 \mathrm{me} 3$ and $\mathrm{H} 3 \mathrm{~K} 27 \mathrm{ac}$ that show a more dispersed pattern in the nucleoplasm. Scale $=5$ um. (D) Coefficient of determination $\left(\mathrm{R}^{2}\right)$ heat map matrix of active histone modifications measured in H3-K27M HEK293 cells. (E) Coefficient of determination $\left(\mathrm{R}^{2}\right)$ heat map matrix of active histone modifications measured in SU-DIPG13 cells. 
Fig. 6

A B

C

bioRxiy preprint doi: https://doi.org/10.1101/2021.11.02.466907; this version posted November 4, 2021. The copyriahth3G9aler for this preprint (whichwas nft certified by peer review) is the author/funder, who has granted bioRxiv a license to display the preprint in perpetuity. It is made

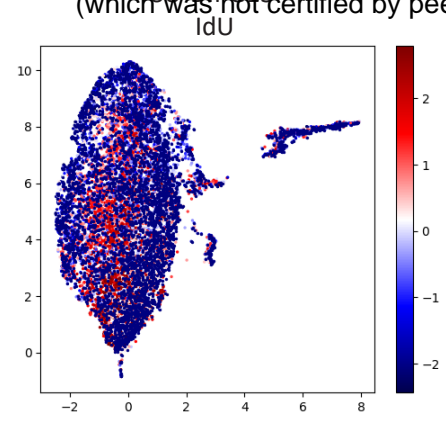

D

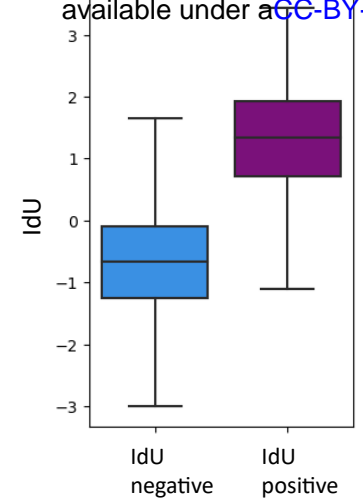

H3K64ac

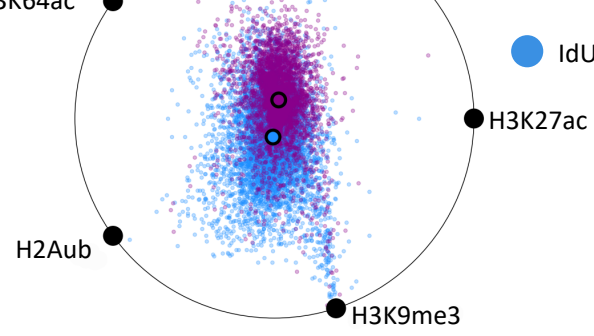

$\mathrm{E}$

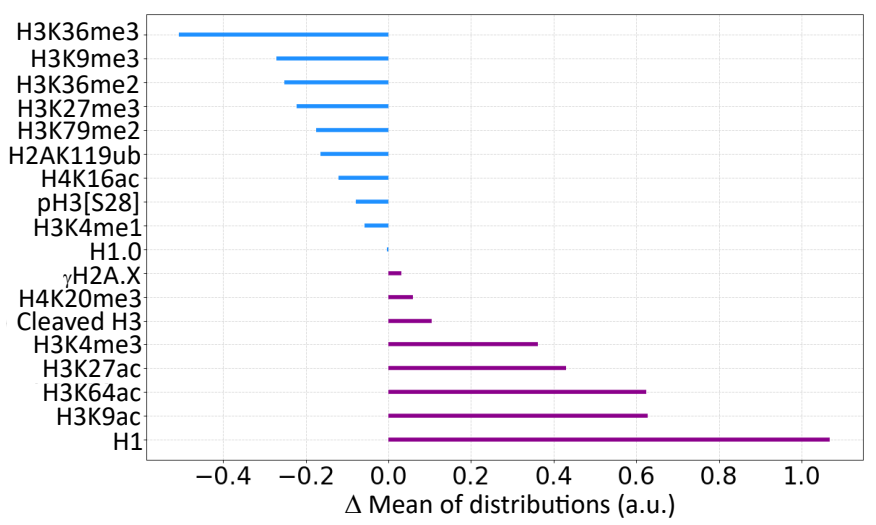

$\mathrm{F}$
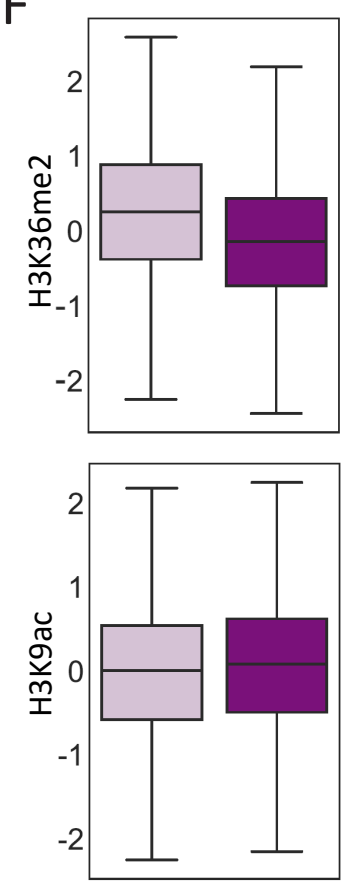
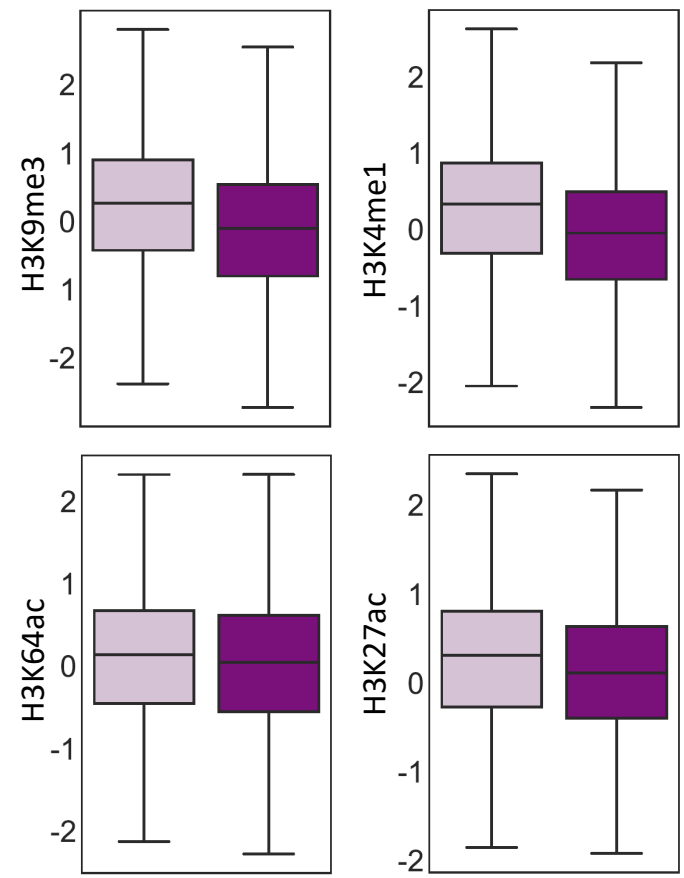
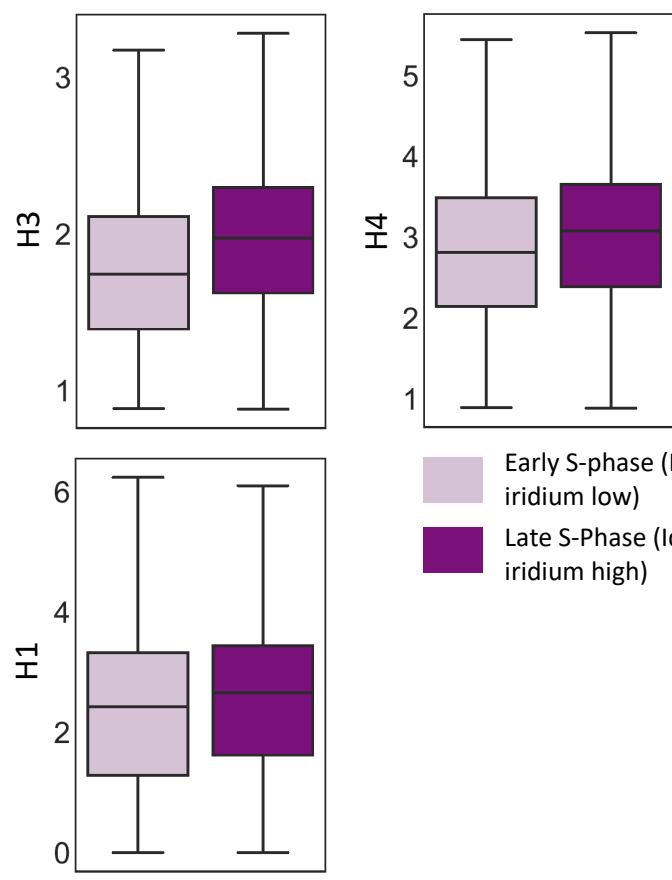

IdU negative is made

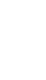




\section{Figure 6: High-dimensional single-cell analysis of epigenetic alterations during cell cycle}

(A) UMAP of SU-DIPG13 as in figure 5a. Shown are the scaled levels of IdU. (B-D) SU-DIPG13

cells were gated based on IdU levels as IdU-positive or IdU-negative. (B) Box plots of IdU scaled levels in the two groups. (C) Graphical representation of the epigenetic state of the two IdU-based cell populations. (D) Fold change differences between the two groups for the indicated epigenetic modifications. Mean values (after transformation, scaling and normalization) of the IdU-negative cells were subtracted from the IdU-positive cells. (E-F) IdU-positive cells were divided to early Sphase and late S-phase based on labeling of total DNA content by iridium. (E) Box plots of the non-normalized scaled expression levels of the core histones during S-phase, indicating an increase in their expression levels. (F) Box plots of the indicated epigenetic modifications normalized to the levels of core histones $\mathrm{H} 3$ and $\mathrm{H} 4$ as measured in $\mathrm{E}$. 